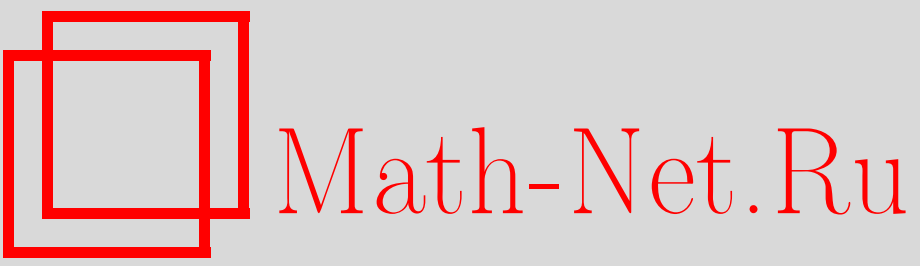

В. А. Краснов, Топологический тип поверхности Фано вещественной трехмерной $M$-кубики, Изв. РАН. Сер. матем., 2005, том 69, выпуск 6, 61-94

DOI: https://doi.org/10.4213/im666

Использование Общероссийского математического портала Math-Net.Ru подразумевает, что вы прочитали и согласны с пользовательским соглашением

http://www.mathnet.ru/rus/agreement

Параметры загрузки:

IP: 18.208 .226 .222

26 апреля 2023 г., 16:01:51 
УДК 512.7

\author{
В. А. Краснов
}

\title{
Топологический тип поверхности Фано вещественной трехмерной $M$-кубики
}

\begin{abstract}
Вычисляется топологический тип вещественной части поверхности Фано, параметризующей множество вещественных прямых на неособом вещественном трехмерном $M$-многообразии третьей степени. При исследовании поверхности Фано используются результаты и конструкции из работы [3] о промежуточном якобиане трехмерной комплексной кубики. Поэтому вначале вычисляется топологический тип вещественной части поверхности Фано, параметризующей множество вещественных прямых на особой вещественной $M$-кубике с единственной простой особой точкой.
\end{abstract}

Библиография: 11 наименований.

\section{Введение}

Далее $V$ - трехмерное комплексное алгебраическое многообразие в комплексном проективном пространстве $\mathbb{P}^{4}$, заданное уравнением

$$
P\left(X_{0}, X_{1}, X_{2}, X_{3}, X_{4}\right)=0
$$

где $P(X)=P\left(X_{0}, X_{1}, X_{2}, X_{3}, X_{4}\right)$ - однородный многочлен третьей степени с вешественными коэффициентами. Будем предполагать, что многообразие $V$ неособое. Через $\mathbb{R} V$ обозначим множество вещественных решений уравнения (0.1), оно всегда непустое и состоит из одной или двух компонент связности. Через $F_{V}=F$ обозначим поверхность $Ф$ ано кубики $X$, которая параметризует множество прямых на $V$. Она будет также неособым многообразием, определенным над полем вешественных точек, так как многочлен $P(X)$ имеет вещественные коэффициенты. Поэтому определено множество вещественных точек $\mathbb{R} F$ комплексной поверхности $\Phi$ ано $F$, параметризуюшее множество прямых на $\mathbb{R} V$. Это множество также всегда непустое, так как на вещественной кубике всегда имеются вещественные прямые. Таким образом, $\mathbb{R} F$ - компактная дифференцируема поверхность. На многообразиях $V, F$ определена инволюция комплексного сопряжения $\tau$, причем множества вешественных точек $\mathbb{R} V, \mathbb{R} F$ совпадают со множествами неподвижных точек этой инволюции: $\mathbb{R} V=V^{\tau}, \mathbb{R} F=F^{\tau}$. Поэтому имеют место неравенства Гарнака-Тома

$$
\begin{aligned}
& \operatorname{dim}_{\mathbb{F}_{2}} H^{*}\left(\mathbb{R} V, \mathbb{F}_{2}\right) \leqslant \operatorname{dim}_{\mathbb{F}_{2}} H^{*}\left(V, \mathbb{F}_{2}\right), \\
& \operatorname{dim}_{\mathbb{F}_{2}} H^{*}\left(\mathbb{R} F, \mathbb{F}_{2}\right) \leqslant \operatorname{dim}_{\mathbb{F}_{2}} H^{*}\left(F, \mathbb{F}_{2}\right),
\end{aligned}
$$


где $H^{*}\left(-, \mathbb{F}_{2}\right)=\bigoplus_{q} H^{q}\left(-, \mathbb{F}_{2}\right)$ и $\mathbb{F}_{2}=\{0,1\}$ - поле из двух элементов. Напомним, что каждое из вещественных многообразий $(V, \tau),(F, \tau)$ называется $M$-многообразием, если соответствуюшее ему неравенство (0.2) или (0.3) становится равенством.

В заметке [9] доказана

Теорема 0.1. Поверхность Фано $(F, \tau)$ будет тогда и только тогда $M$-поверхностью, когда кубика $(V, \tau)$ является $M$-многообразием.

С другой стороны, возникает вопрос о том, как устроена топологическая поверхность $\mathbb{R} F$ в случае, когда вешественная кубика $(V, \tau)$ является $M$-многообразием. Конечно, аналогичный вопрос можно задать и в случае произвольной кубики, но в настоящей статье мы ограничились рассмотрением $M$-кубики, так как этот случай наиболее интересен, а получить результат можно с наименьшими трудностями.

Прежде чем сформулировать соответствуюшую теорему, введем следующие обозначения для топологических поверхностей. Через $S_{g}^{2}$ обозначим связную компактную ориентируемую поверхность без края рода $g$, т.е. топологическую поверхность, гомеоморфную сфере с $g$ ручкам; в частности, $S_{1}^{2}$ - тор, он также обозначается через $T^{2}$. Через $g S^{2}$ обозначим связную компактную неориентируемую поверхность без края рода $g$, т. е. топологическую поверхность, гомеоморфную сфеpe с $g$ пленками Мёбиуса; в частности, ${ }_{1} S^{2}$ - проективная плоскость, она также обозначается через $\mathbb{R} \mathbb{P}^{2}$. Кроме этого, если $S$ - топологическая поверхность, а $n$ натуральное число, то через $n S$ обозначим несвязную сумму $n$ экземпляров поверхности $S$.

В настоящей статье доказывается

Теорема 0.2. Вещественная часть $\mathbb{R} F$ поверхности Фано $F$ неособой $M$-кубики $V$ может имеет только топологический тип ${ }_{5} S^{2} \sqcup 15 T^{2}$.

Сделаем теперь несколько замечаний о структуре данной статьи и о доказательстве теоремы 0.2 .

В $\S 1$ приводятся вспомогательные факты об симметрическом квадрате $C^{(2)}$ комплексной кривой $C$ с вещественной структурой $\tau: C \rightarrow C$. Некоторые из этих фактов являются общеизвестными, а другие, связные с вещественной структурой, являются новыми. Сначала рассматривается симметрический квадрат кривой $(C, \tau)$ произвольного рода $g>0$. Здесь вычисляется вешественная часть $\mathbb{R} C^{(2)}$ симметрического квадрата $C^{(2)}$, а затем доказывается, что поверхность $\left(C^{(2)}, \tau\right)$ будет $M$-поверхностью тогда и только тогда, когда кривая $(C, \tau)$ является $M$-кривой. Затем рассматривается симметрический квадрат канонической $M$-кривой рода 4 . Каждая такая кривая $C$ является неособым полным пересечением квадрики $Q$ и кубики в $\mathbb{P}^{3}$, причем квадрика $Q$ определяется кривой $C$ однозначно. Если квадрика $Q$ неособая, то два семейства прямых на ней порождают два отображения $\lambda^{\prime}, \lambda^{\prime \prime}: C \rightarrow C^{(2)}$, которые являются вложениями (см. п. 1.2). В случае, когда вещественная часть $\mathbb{R} Q$ квадрики $Q$ является гиперболоидом, отображения $\lambda^{\prime}, \lambda^{\prime \prime}$ вешественные, а поэтому определены ограничения этих отображений на вешественные точки $\lambda_{\mathbb{R}}^{\prime}, \lambda_{\mathbb{R}}^{\prime \prime}: \mathbb{R} C \rightarrow \mathbb{R} C^{(2)}$. Мы показываем, что если $(C, \tau)$ $M$-кривая, то отображения $\lambda_{\mathbb{R}}^{\prime}, \lambda_{\mathbb{R}}^{\prime \prime}$ гомотопны. Для доказательства этого утверждения пришлось изучить пространство канонических $M$-кривых рода 4 . В част- 
ности, была доказана связность пространства канонических $M$-кривых на гиперболоидах, связность пространства канонических $M$-кривых на квадратичных конусах, а также связность всего пространства канонических $M$-кривых. Для доказательства этих фактов мы использовали результаты о жесткой изотопической классификации вешественных кривых бистепени $(3,3)$ на гиперболоиде и кубических сечений квадратичного конуса, изложенные в [5], [4].

Результаты, полученные в $\S 1$, применяются для вычисления топологического типа вещественной части $\mathbb{R} F_{0}$ поверхности $Ф$ ано $F_{0}$ для $M$-кубики $V_{0}$, имеющей единственную особенность - простую двойную точку. Этим вычислениям посвящен $\S 2$. Здесь используется схема вычисления топологии комплексной поверхности $\Phi$ ано $F_{0}$ особой кубики $V_{0}$ из работы [3], но только эта схема применяется в вещественной ситуации. Опишем соответствующие конструкции более подробно. Если $p_{0} \in V_{0}$ - особая точка кубики $V_{0} \subset \mathbb{P}^{4}$, то обозначим через $\mathbb{P}^{3}$ проективизацию касательного пространства $T_{p_{0}} \mathbb{P}^{4}$. Таким образом, точками $\mathbb{P}^{3}$ являются прямые в $\mathbb{P}^{4}$, проходящие через $p_{0}$. Проективизация касательного конуса к кубике $V_{0}$ в точке $p_{0}$ дает квадрику $Q \subset \mathbb{P}^{3}$. А множество прямых, лежащих на кубике $V_{0}$ и проходящих через особую точку $p_{0}$, определяет каноническую кривую $C$

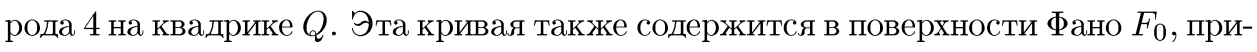
чем множество особых точек поверхности $\Phi$ ано $F_{0}$ совпадает с кривой $C$, которая является простой двойной кривой этой особой поверхности (см. [3]). Мы показываем, что вещественное алгебраическое многообразие $\left(V_{0}, \tau\right)$ тогда и только тогда будет $M$-многообразием, когда вешественная алгебраическая кривая $(C, \tau)$ является $M$-кривой.

Если теперь $C^{\prime}, C^{\prime \prime} \subset C^{(2)}$ - образы кривой $C$ при отображениях $\lambda^{\prime}, \lambda^{\prime \prime}$, то поверхность Фано $F_{0}$ получается из симметрического квадрата $C^{(2)}$ с помощью отождествления кривых $C^{\prime}, C^{\prime \prime} \subset C^{(2)}$, которое производится отображением $\lambda^{\prime \prime}$ 。 $\left(\lambda^{\prime}\right)^{-1}: C^{\prime} \rightarrow C^{\prime \prime}$, причем из кривых $C^{\prime}, C^{\prime \prime} \subset C^{(2)}$ получается двойная кривая $C \subset F_{0}[3]$. Из этого результата сразу выводится, что если квадрика $\mathbb{R} Q$ является эллипсоидом, то вешественная часть $\mathbb{R} F_{0}$ поверхности Фано $F_{0}$ гомеоморфна несвязной сумме $\mathbb{R} C^{(2)} \sqcup \mathbb{R} C$.

Если квадрика $\mathbb{R} Q$ является гиперболоидом, то задача вычисления топологического типа поверхности $\mathbb{R} F_{0}$ оказывается более сложной. В настоящей статье мы решаем ее только в случае, когда $(C, \tau)-M$-кривая. Сначала заметим, что особая поверхность $\mathbb{R} F_{0}$ получается из неособой поверхности $\mathbb{R} C^{(2)}$ с помошью отождествления кривых $\mathbb{R} C^{\prime}, \mathbb{R} C^{\prime \prime}$, которое производится отображением

$$
\lambda_{\mathbb{R}}^{\prime \prime} \circ\left(\lambda_{\mathbb{R}}^{\prime}\right)^{-1}: \mathbb{R} C^{\prime} \rightarrow \mathbb{R} C^{\prime \prime}
$$

Затем, используя гомотопность отображений $\lambda_{\mathbb{R}}^{\prime}, \lambda_{\mathbb{R}}^{\prime \prime}$, а также мономорфность гомоморфизмов

$$
\left(\lambda_{\mathbb{R}}^{\prime}\right)_{*},\left(\lambda_{\mathbb{R}}^{\prime \prime}\right)_{*}: H_{*}\left(\mathbb{R} C, \mathbb{F}_{2}\right) \rightarrow H_{*}\left(\mathbb{R} C^{(2)}, \mathbb{F}_{2}\right),
$$

удается описать топологический тип особой поверхности $\mathbb{R} F_{0}$ (см. предложение 2.3). Сделаем несколько замечаний об этом топологическом типе.

Прежде всего заметим, что вешественная часть $\mathbb{R} C^{(2)}$ симметрического квадрата $C^{(2)}$ имеет топологический тип ${ }_{5} S^{2} \sqcup 10 T^{2}$. Далее неориентируемую компоненту ${ }_{5} S^{2}$ этой поверхности будем обозначать через П. На поверхности П расположены по одной из компонент связности кривых $\mathbb{R} C^{\prime}, \mathbb{R} C^{\prime \prime}$, обозначим эти 
компоненты через $\Gamma_{0}^{\prime}, \Gamma_{0}^{\prime \prime}$ соответственно. Эти компоненты разбивают поверхность П на две поверхности с краем: $\Pi^{\prime}, \Pi^{\prime \prime}$, первая из которых является цилиндром, а вторая - неориентируемой поверхностью рода 3 с двумя дырами. После склейки краев из поверхностей $\Pi^{\prime}, \Pi^{\prime \prime}$ получаются топологические поверхности без края $\Pi_{1}, \Pi_{2}$, первая из которых является тором, а вторая - неориентируемой поверхностью рода 5 . Поверхности $\Pi_{1}, \Pi_{2}$ пересекаются по кривой $\Gamma_{0} \subset \mathbb{R} C$, которая получается из компонент $\Gamma_{0}^{\prime}, \Gamma_{0}^{\prime \prime}$ после их отождествления.

Кроме поверхности П вешественная часть $\mathbb{R} C^{(2)}$ симметрического квадрата $C^{(2)}$ содержит 10 торов. На четырех из них $T_{1}, \ldots, T_{4}$ содержатся по одной из оставшихся четырех компонент кривых $\mathbb{R} C^{\prime}, \mathbb{R} C^{\prime \prime}$, обозначим эти компоненты через $\Gamma_{1}^{\prime}, \Gamma_{1}^{\prime \prime}, \ldots, \Gamma_{4}^{\prime}, \Gamma_{4}^{\prime \prime}$. Итак, $\Gamma_{1}^{\prime}, \Gamma_{1}^{\prime \prime} \subset T_{1}, \ldots, \Gamma_{4}^{\prime}, \Gamma_{4}^{\prime \prime} \subset T_{4}$. Каждая пара кривых $\Gamma_{i}^{\prime}, \Gamma_{i}^{\prime \prime} \subset T_{i}, i=1, \ldots, 4$, разбивает тор $T_{i}$ на два цилиндра $\Pi_{i}^{\prime}, \Pi_{i}^{\prime \prime}$. После склейки краев этих цилиндров из них получаются топологические торы $T_{i}^{\prime}, T_{i}^{\prime \prime}$, которые пересекаются по кривой $\Gamma_{i} \subset \mathbb{R} C$, полученной из компонент $\Gamma_{i}^{\prime}, \Gamma_{i}^{\prime \prime}$ после их отождествления.

В конще $\S 2$ доказывается, что если вешественная алгебраическая кривая $(C, \tau)$ является $M$-кривой, то вещественная алгебраическая поверхность $\left(F_{0}, \tau\right)$ будет $M$-поверхностью.

В начале $\S 3$ приводится набросок доказательства теоремы 0.1. Эта теорема используется при доказательстве теоремы 0.2 , поэтому для удобства читателя изложены основные идеи ее доказательства. В $\S 3$ с помощью результатов первых двух параграфов вычисляется топологический тип вешественной части $\mathbb{R} F$ поверхности $\Phi$ ано $F$ неособой $M$-кубики $V$, т. е. доказывается теорема 0.2 . Попутно в этом параграфе доказывается существование неособых $M$-кубик. Здесь используется схема вычисления топологии комплексной поверхности Фано $F$ неособой кубики $V[3]$, но только эта схема применяется в вещественной ситуации. Изложим кратко соответствующую конструкцию.

Данная неособая кубика $V$ может быть включена в линейное семейство кубик $V_{t}, t \in \mathbb{P}^{1}$, которое представляет собой пучок гиперплоских сечений неособой четырехмерной кубики $Z \subset \mathbb{P}^{5}$, причем особые сечения содержат только одну простую двойную точку, а других особых точек нет. Это семейство образовано сечениями гиперплоскостей, проходящих через фиксированное трехмерное проективное пространство $R \subset \mathbb{P}^{5}$. Если исходная кубика $V$ вешественная, то существует аналогичное вещественное линейное семейство $V_{t}$, содержащее данную кубику. Это означает, что оно определено над полем вещественных чисел, а поэтому имеется отображение комплексного сопряжения $\tau: V_{t} \rightarrow V_{\tau(t)}$. В частности, если точка $t$ вешественная, т.е. $t \in \mathbb{R P}^{1}$, то кубика $V_{t}$ вещественная, а также справедливо обратное утверждение. Мы можем считать, что при $t=1$ получаем исходную неособую кубику $V$, а ближайшая вешественная особая кубика получается при $t=0$, где $t$ - аффинная координата на $\mathbb{P}^{1}$. Выберем такое малое число $0<\varepsilon<1$, чтобы многообразия $V_{t}$ были неособые при $0<|t|<2 \varepsilon$. Заметим, что многообразия $\left(V_{1}, \tau\right),\left(V_{\varepsilon}, \tau\right)$ эквивариантно диффеоморфны. Поэтому кубика $\left(V_{1}, \tau\right)$ будет тогда и только тогда $M$-многообразием, когда такой является кубика $\left(V_{\varepsilon}, \tau\right)$. Семейство кубик $V_{t}$ порождает семейство поверхностей Фано $F_{t}$, причем если кубика $V_{t}$ неособая, то и поверхность $\Phi$ ано $F_{t}$ неособая. Поэтому поверхности $Ф$ ано $\left(F_{1}, \tau\right),\left(F_{\varepsilon}, \tau\right)$ эквивариантно диффеоморфны. Следовательно, теорему 0.2 достаточно доказать 
для кубики $\left(V_{\varepsilon}, \tau\right)$. Обозначим кубики $V_{ \pm \varepsilon}$ кратко через $V_{ \pm}$. Мы показываем, что если неособая кубика $\left(V_{+}, \tau\right)$ является $M$-многообразием, то особая кубика $\left(V_{0}, \tau\right)$ также будет $M$-многообразием. Доказательство этого утверждения несложное; оказывается, что более трудно доказать аналог обратного утверждения: если особая кубика $\left(V_{0}, \tau\right)$ является $M$-многообразием, то одна из неособых кубик $\left(V_{ \pm}, \tau\right)$ будет $M$-многообразием, а другая не будет. Заметим, что из этого утверждения вытекает существование неособых $M$-кубик.

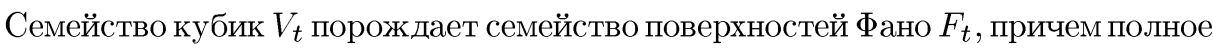
тело этого семейства

$$
\mathfrak{F}=\bigcup_{t \in \mathbb{P}^{1}} F_{t}
$$

неособое (см. [3]). Рассмотрим соответствующую функцию $f: \mathfrak{F} \rightarrow \mathbb{P}^{1}$, которая отображает поверхность $F_{t}$ в точку $t \in \mathbb{P}^{1}$. Ограничим эту функцию на вешественные точки $f_{\mathbb{R}}: \mathbb{R} \mathfrak{F} \rightarrow \mathbb{R} \mathbb{P}^{1}$. Эта функция не является функцией Морса, так как все точки из кривой $\mathbb{R} C \subset \mathbb{R} F_{0}$ критические. Однако нормальное расслоение к кривой $\mathbb{R} C$ в $\mathbb{R} \mathfrak{F}$ тривиально, а кривая $C$ является простой двойной кривой на $F_{0}$, поэтому окрестность кривой $\mathbb{R} C$ в $\mathbb{R} \mathfrak{F}$ является тривиальным расслоением на диски, причем на каждом из этих дисков функция $f_{\mathbb{R}}$ имеет невырожденную особую точку. Необходимо показать, что $\mathbb{R} F_{\varepsilon}=\left(f_{\mathbb{R}}\right)^{-1}(\varepsilon)$ имеет топологический тип ${ }_{5} S^{2} \sqcup 15 T^{2}$, а мы уже знаем, как устроена особая поверхность $\mathbb{R} F_{0}=\left(f_{\mathbb{R}}\right)^{-1}(0)$. Тогда неособая поверхность $\mathbb{R} F_{\varepsilon}$ получается из особой $\mathbb{R} F_{0}$ с помощью стандартных перестроек, причем из максимальности поверхности $\left(F_{\varepsilon}, \tau\right)$ вытекает, что такие перестройки однозначно определены. Оказывается, что в случае, когда квадрика $\mathbb{R} Q$ является эллипсоидом, перестройки поверхности $\mathbb{R} F_{0}=\mathbb{R} C^{(2)} \sqcup \mathbb{R} C$ устроены следующим образом: компоненты из $\mathbb{R} C^{(2)}$ не меняются, а каждая компонента кривой $\mathbb{R} C$ заменяется на тор. В случае, когда квадрика $\mathbb{R} Q$ является гиперболоидом, эти перестройки разводят поверхности $\Pi_{1}, \Pi_{2}$, a также торы $T_{i}^{\prime}, T_{i}^{\prime \prime}$, $i=1, \ldots, 4$, т. е. делают их непересекающимися, а неособые компоненты поверхности $\mathbb{R} F_{0}$ не меняются. Следовательно, в каждом случае поверхность $\mathbb{R} F_{\varepsilon}$ имеет топологический тип ${ }_{5} S^{2} \sqcup 15 T^{2}$.

\section{§1. Симметрический квадрат кривой}

Пусть $C$ - связная компактная риманова поверхность рода $g>0$, а $C^{(2)}-$ ее симметрический квадрат. Вычислим группы когомологий комплексной поверхности $C^{(2)}$. Прежде всего заметим, что группа $H^{*}\left(C^{(2)}, \mathbb{Z}\right)$ свободная (см., например, [9]). Пусть $\sigma: C^{2} \rightarrow C^{2}$ - инволюция, переставляющая сомножители. Тогда $C^{(2)}=C^{2} / \sigma-$ факторпространство, поэтому выполняется равенство для групп когомологий с рациональными коэффициентами

$$
H^{p}\left(C^{(2)}, \mathbb{Q}\right)=H^{p}\left(C^{2}, \mathbb{Q}\right)^{\sigma},
$$

где $H^{p}\left(C^{2}, \mathbb{Q}\right)^{\sigma}$ - подпространство инвариантных относительно $\sigma$ элементов из $H^{p}\left(C^{2}, \mathbb{Q}\right)$. Группа $H^{1}\left(C^{2}, \mathbb{Q}\right)$ состоит из сумм $p_{1}^{*}\left(a_{1}\right)+p_{2}^{*}\left(a_{2}\right)$, где $a_{1}, a_{2} \in$ 
$H^{1}(C, \mathbb{Q}), \quad p_{1}, p_{2}: C^{2} \rightarrow C$ - проекции. Тогда инволюция $\sigma^{*}: H^{1}\left(C^{2}, \mathbb{Q}\right) \rightarrow$ $H^{1}\left(C^{2}, \mathbb{Q}\right)$ действует по правилу

$$
\sigma^{*}\left(p_{1}^{*}\left(a_{1}\right)+p_{2}^{*}\left(a_{2}\right)\right)=p_{2}^{*}\left(a_{1}\right)+p_{1}^{*}\left(a_{2}\right)
$$

Из этого равенства вытекает, что для поверхности $C^{(2)}$ имеем при $p=1,3$ равенства

$$
\operatorname{dim} H^{p}\left(C^{(2)}, \mathbb{F}_{2}\right)=\operatorname{dim} H^{p}\left(C^{(2)}, \mathbb{Q}\right)=2 g .
$$

Группа $H^{2}\left(C^{2}, \mathbb{Q}\right)$ состоит из сумм

$$
p_{1}^{*}\left(a_{1}\right)+p_{2}^{*}\left(a_{2}\right)+\sum_{i} p_{1}^{*}\left(b_{i}^{\prime}\right) \cup p_{2}^{*}\left(b_{i}^{\prime \prime}\right)
$$

где $a_{1}, a_{2} \in H^{2}(C, \mathbb{Q}), b_{i}^{\prime}, b_{i}^{\prime \prime} \in H^{1}(C, \mathbb{Q})$, а инволюция $\sigma^{*}: H^{2}\left(C^{2}, \mathbb{Q}\right) \rightarrow H^{2}\left(C^{2}, \mathbb{Q}\right)$ действует по правилу

$\sigma^{*}\left(p_{1}^{*}\left(a_{1}\right)+p_{2}^{*}\left(a_{2}\right)+\sum_{i} p_{1}^{*}\left(b_{i}^{\prime}\right) \cup p_{2}^{*}\left(b_{i}^{\prime \prime}\right)\right)=p_{2}^{*}\left(a_{1}\right)+p_{1}^{*}\left(a_{2}\right)+\sum_{i} p_{2}^{*}\left(b_{i}^{\prime}\right) \cup p_{1}^{*}\left(b_{i}^{\prime \prime}\right)$.

Следовательно,

$$
\operatorname{dim} H^{2}\left(C^{(2)}, \mathbb{F}_{2}\right)=\operatorname{dim} H^{2}\left(C^{(2)}, \mathbb{Q}\right)=2 g^{2}-g+1
$$

1.1. Симметрический квадрат $M$-кривой. Если на комплексной кривой $C$ определена вещественная структура, т. е. антиголоморфная инволюция $\tau: C \rightarrow C$, то на симметрическом квадрате $C^{(2)}$ индуцируется вещественна структура по правилу

$$
\tau\left(p_{1}+p_{2}\right)=\tau\left(p_{1}\right)+\tau\left(p_{2}\right)
$$

где точка на $C^{(2)}$ записьвается в виде формальной суммы $p_{1}+p_{2}$ двух точек $p_{1}, p_{2}$ из кривой $C$. Множество вешественных точек $\mathbb{R} C$ комплексной кривой $C$ представляет собой, в терминах топологии, несвязное объединение $k$ окружностей, где число $k$ удовлетворяет неравенству Гарнака

$$
k \leqslant g+1
$$

При $k=g+1$ кривая $C$ называется $M$-кривой. В этом пункте мы опишем топологическое строение множества вешественных точек $\mathbb{R} C^{(2)}$ симметрического квадpaтa $C^{(2)}$.

Рассмотрим сначала случай, когда множество $\mathbb{R} C$ пустое. Тогда множество $\mathbb{R} C^{(2)}$ состоит из сумм вида $p+\tau(p)$, где $p-$ произвольная точка кривой $C$. Следовательно, в этом случае множество вешественных точек $\mathbb{R} C^{(2)}$ гомеоморфно неориентируемой факторповерхности $\bar{C}=C / \tau$ и выполняется равенство

$$
\operatorname{dim} H^{1}\left(\mathbb{R} C^{(2)}, \mathbb{F}_{2}\right)=g+1
$$

т. е. $\mathbb{R} C^{(2)}={ }_{g+1} S^{2}$. 
Рассмотрим теперь случай, когда множество $\mathbb{R} C$ непустое. Пусть оно состоит из топологических окружностей $\Sigma_{1}, \ldots, \Sigma_{k}$. Тогда множество вещественных точек $\mathbb{R} C^{2}$ декартова квадрата $C^{2}$ представляет собой несвязную сумму торов $\Sigma_{i} \times \Sigma_{j}$, где $1 \leqslant i, j \leqslant k$. Симметрический квадрат $C^{(2)}$ кривой $C$ получается из декартова квадрата $C^{2}$ с помощью факторизации по инволюции $\sigma: C^{2} \rightarrow C^{2}$, переставляющей сомножители. Пусть $i<j$, тогда после факторизации два тора $\Sigma_{i} \times \Sigma_{j}, \quad \Sigma_{j} \times \Sigma_{i}$ отождествляются в один тор, который будем обозначать через $T_{i j}$. Торы $T_{i j}, i<j$, будут входить во множество вещественных точек $\mathbb{R} C^{(2)}$ симметрического квадрата $C^{(2)}$. Из каждого тора $\Sigma_{i} \times \Sigma_{i}, i=1, \ldots, k$, после факторизации получается лист Мёбиуса $M_{i}$. Эти листы Мёбиуса также входят во множество $\mathbb{R} C^{(2)}$. Кроме того, в него входят точки вида $p+\tau(p)$, которые образуют множество, гомеоморфное факторповерхности $\bar{C}=C / \tau$. Она представляет собой поверхность с краем, причем край состоит из $k$ компонент, каждая из которых является краем одного листа Мёбиуса $M_{i}$. Таким образом, объединение $\Pi=\bar{C} \cup M_{1} \cup \ldots \cup M_{k}$ представляет собой связную компактную дифференцируемую поверхность, которая гомеоморфна поверхности, полученной из факторповерхности $\bar{C}=C / \tau$ с помощью заклеивания дыр листами Мёбиуса. Поверхность П неориентируема и гомеоморфна $g+1 S^{2}$. В итоге получаем, что множество вешественных точек $\mathbb{R} C^{(2)}$ в случае, когда множество $\mathbb{R} C$ состоит из $k$ компонент, представляет собой объединение:

$$
\mathbb{R} C^{(2)}=\bigcup_{i<j} T_{i j} \cup \Pi
$$

где П - неориентируемая поверхность рода $g+1$ без края, т. е. $\Pi={ }_{g+1} S^{2}$. Из этого соотношения вытекает равенство

$$
\operatorname{dim} H^{*}\left(\mathbb{R} C^{(2)}, \mathbb{F}_{2}\right)=2 k(k-1)+g+3,
$$

которое выполняется и в случае пустого множества $\mathbb{R} C$. С другой стороны, имеем

$$
\operatorname{dim} H^{*}\left(C^{(2)}, \mathbb{F}_{2}\right)=2 g^{2}+3 g+3
$$

Таким образом, только при $k=g+1$ вьполняется равенство

$$
\operatorname{dim} H^{*}\left(\mathbb{R} C^{(2)}, \mathbb{F}_{2}\right)=\operatorname{dim} H^{*}\left(C^{(2)}, \mathbb{F}_{2}\right) .
$$

Тем самым доказано

ПРЕДЛОЖЕНИЕ 1.1. Симметрический квадрат $\left(C^{(2)}, \tau\right)$ будет тогда $u$ только тогда $M$-многообразием, когда кривая $(C, \tau)$ является $M$-кривой. 
1.2. Симметрический квадрат канонической кривой рода 4. Напомним, что неособая кривая $C \subset \mathbb{P}^{3}$ рода 4 назьвается канонической, если существует базис голоморфных форм $\omega_{1}, \ldots, \omega_{4}$ на $C$ такой, что отображение вложения $C \hookrightarrow \mathbb{P}^{3}$ задается равенством

$$
\left[Y_{1}, \ldots, Y_{4}\right]=\left[\omega_{1}(p), \ldots, \omega_{4}(p)\right]
$$

где $Y_{1}, \ldots, Y_{4}$ - однородные координаты в $\mathbb{P}^{3}, p$-переменная точка на $C$. Каждая такая кривая является неособым полным пересечением квадрики и кубики в $\mathbb{P}^{3}$, причем квадрика определяется кривой $C$ однозначно, будем ее обозначать через $Q(C)$. Также верно обратное утверждение. Таким образом, степень канонической кривой в $\mathbb{P}^{3}$ равна 6 . Обозначим через $\mathcal{H}_{6,4,3}$ схему Гильберта кривых в $\mathbb{P}^{3}$ степени 6 и рода 4 . Тогда множество канонических кривых образует на схеме Гильберта открытое множество в топологии Зариского, которое будем обозначать через $\mathcal{C}$. Таким образом, $\mathcal{C}$ - это пространство канонических кривых рода 4 в $\mathbb{P}^{3}$. Все точки из $\mathcal{C}$ неособые. Это вытекает из равенства $H^{1}(C, \mathcal{N})=0$, где $\mathcal{N}$ - пучок ростков нормального расслоения к канонической кривой $C$ в $\mathbb{P}^{3}$.

Пусть $Q$ - квадрика в трехмерном комплексном проективном пространстве $\mathbb{P}_{Y}^{3}$ с однородными координатами $Y_{1}, \ldots, Y_{4}$. Тогда она определяется уравнением

$$
q(Y)=\sum_{i, j=1}^{4} a_{i j} Y_{i} Y_{j}=0,
$$

где $A_{Q}=\left(a_{i j}\right)$ - ненулевая симметрическая матрица размера $4 \times 4$. Пространство таких квадрик обозначим через $\mathcal{Q}$. Коэффициенты матрицы $A_{Q}$ определяют изоморфизм $\mathcal{Q} \cong \mathbb{P}_{W}^{9}$, где $\mathbb{P}_{W}^{9}$ - проективное пространство, ассоциированное с векторным пространством симметрических матриц $A$ размера $4 \times 4$, а $W_{i j}$ - однородные координаты в нем, причем предполагается, что выполняется равенство $W_{j i}=W_{i j}$. Обозначим через $\mathcal{Q}_{k}$ пространство квадрик $Q$, для которых ранг матрищы $A_{Q}$ paвен $k$. Тогда имеем разложение на непересекаюшиеся неособые квазипроективные многообразия:

$$
\mathcal{Q}=\mathcal{Q}_{4} \cup \mathcal{Q}_{3} \cup \mathcal{Q}_{2} \cup \mathcal{Q}_{1}
$$

Здесь $\mathcal{Q}_{4}$ - пространство неособых квадрик, $\mathcal{Q}_{3}$ - пространство квадратичных конусов, $\mathcal{Q}_{2}$ - пространство пар плоскостей, $\mathcal{Q}_{1}$ - пространство двойных плоскостей. Объединение $\mathcal{Q}_{4} \cup \mathcal{Q}_{3}$ будем обозначать через $\mathcal{Q}_{\geqslant 3}$, оно является открытым множеством в топологии Зариского на пространстве всех квадрик $\mathcal{Q}=\mathbb{P}_{W}^{9}$.

Правило $C \mapsto Q(C)$ задает регулярное отображение $\pi: \mathcal{C} \rightarrow \mathcal{Q}$. Образ этого отображения состоит из квадрик $Q$, для которых соответствуюшая квадратична форма $q(Y)$ имеет ранг не менее 3 , т.е. $\pi(\mathcal{C})=\mathcal{Q}_{\geqslant 3}$. Прообраз $\pi^{-1}(Q)$, где $Q \in \mathcal{Q}_{\geqslant 3}$, является открытым множеством в топологии Зариского в 15 -мерном проективном пространстве $\mathbb{P}\left(\Gamma\left(Q, \mathcal{O}_{Q}(3)\right)\right)$. На самом деле, многообразие $\mathcal{C}$ является открытым множеством в топологии Зариского расслоения со слоем $\mathbb{P}^{15}$ на пространстве квадрик $\mathcal{Q}_{\geqslant 3}$, которое мы сейчас опишем. В произведении $\left(\mathcal{Q}_{\geqslant 3}\right) \times \mathbb{P}_{Y}^{3}$ полное тело квадрик

$$
\mathfrak{Q}=\bigcup_{w \in \mathcal{Q} \geqslant 3} Q \times w
$$


где $Q \subset \mathbb{P}_{Y}^{3}-$ квадрика, отвечающая точке $w \in \mathcal{Q} \geqslant 3$, образует неособую гиперповерхность. Пусть $f: \mathfrak{Q} \rightarrow \mathcal{Q}_{\geqslant 3}$ - проекция. Тогда пучок $\mathcal{E}=f_{*}\left(\mathcal{O}_{\mathfrak{Q}}(3)\right)$ локально свободный, поэтому имеем локально тривиальное расслоение $\pi: \mathbb{P}(\mathcal{E}) \rightarrow \mathcal{Q}_{\geqslant 3}$ со слоем $\mathbb{P}^{15}$. Многообразие канонических кривых $\mathcal{C}$ является открытым множеством в топологии Зариского в $\mathbb{P}(\mathcal{E})$.

Пусть каноническая кривая $C$ лежит на неособой квадрике $Q$; тогда через каждую точку $p \in C$ проходят две прямые $L_{p}^{\prime}, L_{p}^{\prime \prime}$ из двух семейств прямых на квадрике $Q$. Заметим, что индексы пересечения $\left(C, L_{p}^{\prime}\right),\left(C, L_{p}^{\prime \prime}\right)$ равны 3 , т. е. кривая $C$ на квадрике $Q$ имеет бистепень $(3,3)$. Поэтому определены канонические отображения

$$
\lambda^{\prime}: C \rightarrow C^{(2)}, \quad \lambda^{\prime \prime}: C \rightarrow C^{(2)},
$$

которые устроены следуюшим образом. Прямые $L_{p}^{\prime}, L_{p}^{\prime \prime}$ имеют с кривой $C$, кроме общей точки $p$, еще по две точки (с учетом кратности пересечений), т. е. для циклов пересечения имеем равенства

$$
C \cdot L_{p}^{\prime}=p+p_{1}^{\prime}+p_{2}^{\prime}, \quad C \cdot L_{p}^{\prime \prime}=p+p_{1}^{\prime \prime}+p_{2}^{\prime \prime} .
$$

Тогда по определению полагаем

$$
\lambda^{\prime}(p)=p_{1}^{\prime}+p_{2}^{\prime}, \quad \lambda^{\prime \prime}(p)=p_{1}^{\prime \prime}+p_{2}^{\prime \prime} .
$$

Образы отображений (1.1) обозначим через $C^{\prime}, C^{\prime \prime}$ соответственно. Тогда кривые $C^{\prime}, C^{\prime \prime}$ не пересекаются и отображения

$$
\lambda^{\prime}: C \rightarrow C^{\prime}, \quad \lambda^{\prime \prime}: C \rightarrow C^{\prime \prime}
$$

являются изоморфизмами комплексных алгебраических кривых.

На конусе $Q_{0}$ имеется только одно семейство прямых $L_{p}$. Оно задает отображение

$$
\lambda_{0}: C_{0} \rightarrow\left(C_{0}\right)^{(2)},
$$

причем отображение на образ $\lambda_{0}: C_{0} \rightarrow \lambda_{0}\left(C_{0}\right)$ является изоморфизмом комплексных алгебраических кривых.

Пусть $C_{t}, 0 \leqslant t \leqslant 1,-$ дифференцируемое семейство канонических кривых, где $Q\left(C_{t}\right) \in \mathcal{Q}_{4}$ при $t>0$ и $Q\left(C_{0}\right) \in \mathcal{Q}_{3}$. Тогда получаем два дифференщируемых семейства регулярных отображений:

$$
\lambda_{t}^{\prime}: C_{t} \rightarrow\left(C_{t}\right)^{(2)}, \quad \lambda_{t}^{\prime \prime}: C_{t} \rightarrow\left(C_{t}\right)^{(2)},
$$

которые совпадают с отображением $\lambda_{0}$ при $t=0$.

ПРЕДЛОЖенИЕ 1.2. Пусть каноническая кривая $C$ лежит на неособой квадрике $Q$. Тогда отображения (1.1) гомотопны в классе дифференцируемых отображений. 
ДокАЗАТЕЛЬСтво. Выберем в пространстве канонических кривых $\mathcal{C}$ дифференцируемый путь $C_{t}, 0 \leqslant t \leqslant 1$, где $C_{1}=C, Q\left(C_{t}\right) \in \mathcal{Q}_{4}$ при $t>0$ и $Q\left(C_{0}\right) \in \mathcal{Q}_{3}$. Такой путь сушествует, так как пространство канонических кривых $\mathcal{C}$ связное. Семейство канонических кривых $C_{t}$ задает тривиальное дифференцируемое расслоение над отрезком $[0,1]$ со слоем $C$. Зафиксируем тривиализацию $c_{t}: C_{t} \rightarrow C$ этого расслоения, где $c_{1}: C_{1}=C \rightarrow C$ - тождественное отображение. Это семейство диффеоморфизмов определяет семейство диффеоморфизмов симметрических квадратов $\left(c_{t}\right)^{(2)}:\left(C_{t}\right)^{(2)} \rightarrow C^{(2)}$. Рассмотрим теперь два дифференцируемых семейства дифференцируемых отображений:

$$
\Lambda_{t}^{\prime}=\left(c_{t}\right)^{(2)} \circ \lambda_{t}^{\prime} \circ c_{t}^{-1}: C \rightarrow C^{(2)}, \quad \Lambda_{t}^{\prime \prime}=\left(c_{t}\right)^{(2)} \circ \lambda_{t}^{\prime \prime} \circ c_{t}^{-1}: C \rightarrow C^{(2)},
$$

где $\lambda_{0}^{\prime}=\lambda_{0}^{\prime \prime}=\lambda_{0}$. Эти семейства отображений задают гомотопии отображения

$$
\Lambda_{0}=\left(c_{0}\right)^{(2)} \circ \lambda_{0} \circ c_{0}^{-1}: C \rightarrow C^{(2)}
$$

с отображениями (1.1). Предложение доказано.

Пусть $Q(C) \in \mathcal{Q}_{4}$ и $c \in C$ - фиксированная точка. Тогда рассмотрим отображения Абеля-Якоби

$$
\begin{aligned}
& \alpha: C \rightarrow J, \quad \alpha(p)=[p-c], \\
& \alpha^{(2)}: C^{(2)} \rightarrow J, \quad \alpha^{(2)}\left(p_{1}+p_{2}\right)=\alpha\left(p_{1}\right)+\alpha\left(p_{2}\right)=\left[p_{1}+p_{2}-2 c\right],
\end{aligned}
$$

где $[p-c],\left[p_{1}+p_{2}-2 c\right] \in J-$ классы линейной эквивалентности дивизоров нулевой степени на кривой $C$, а многообразие Якоби $J$ отождествляется с многообразием Пикара $\operatorname{Pic}^{0}(C)$. Композиции отображений

$$
\alpha^{(2)} \circ \lambda^{\prime}: C \rightarrow J, \quad \alpha^{(2)} \circ \lambda^{\prime \prime}: C \rightarrow J
$$

будем обозначать через $\alpha^{\prime}, \alpha^{\prime \prime}$ соответственно.

Если выполняются равенства для дивизоров

$$
C \cdot L_{c}^{\prime}=c+c_{1}^{\prime}+c_{2}^{\prime}, \quad C \cdot L_{c}^{\prime \prime}=c+c_{1}^{\prime \prime}+c_{2}^{\prime \prime}
$$

то переменный дивизор $p+p_{1}^{\prime}+p_{2}^{\prime}$ на $C$ линейно эквивалентен дивизору $c+c_{1}^{\prime}+c_{2}^{\prime}$, а дивизор $p+p_{1}^{\prime \prime}+p_{2}^{\prime \prime}$ линейно эквивалентен дивизору $c+c_{1}^{\prime \prime}+c_{2}^{\prime \prime}$. Поэтому выполняются равенства для классов линейной эквивалентности дивизоров

$$
\begin{aligned}
{\left[p_{1}^{\prime}+p_{2}^{\prime}-2 c\right] } & =[c-p]+\left[c_{1}^{\prime}+c_{2}^{\prime}-2 c\right], \\
{\left[p_{1}^{\prime \prime}+p_{2}^{\prime \prime}-2 c\right] } & =[c-p]+\left[c_{1}^{\prime \prime}+c_{2}^{\prime \prime}-2 c\right] .
\end{aligned}
$$

Следовательно, для отображений $\alpha^{\prime}: C \rightarrow J, \alpha^{\prime \prime}: C \rightarrow J$ справедливы равенства

$$
\alpha^{\prime}(p)=-\alpha(p)+\alpha^{\prime}(c), \quad \alpha^{\prime \prime}(p)=-\alpha(p)+\alpha^{\prime \prime}(c) .
$$

Из этих равенств вытекает 
ПРЕДЛОЖЕНИЕ 1.3. Пусть $A$ - абелева группа; тогда при каждом q отображения групп гомологий

$$
\left(\alpha^{\prime}\right)_{*}: H_{q}(C, A) \rightarrow H_{q}(J, A), \quad\left(\alpha^{\prime \prime}\right)_{*}: H_{q}(C, A) \rightarrow H_{q}(J, A)
$$

совпадают и равнь гомоморфизму $(-\alpha)_{*}: H_{q}(C, A) \rightarrow H_{q}(J, A)$, a поэтому мономорфны.

Применим предложение 1.2 , тогда получим

СЛЕДСТВИЕ 1.4. При каждом q отображения групп гомологий

$$
\left(\lambda^{\prime}\right)_{*}: H_{q}(C, A) \rightarrow H_{q}\left(C^{(2)}, A\right), \quad\left(\lambda^{\prime \prime}\right)_{*}: H_{q}(C, \mathbb{Z}) \rightarrow H_{q}\left(C^{(2)}, A\right)
$$

совпадают и мономорфнье.

В случае, когда каноническая кривая $C$ лежит на квадратичном конусе $Q_{0}$, имеем одно отображение $\alpha_{0}: C \rightarrow J$, которое равно композищии $\alpha^{(2)} \circ \lambda_{0}$. Для него справедливо равенство

$$
\alpha_{0}(p)=-\alpha(p)+\alpha_{0}(c)
$$

Из данного равенства вытекает

ПРЕДЛОЖЕНИЕ 1.5. Если кривая $C$ лежит на квадратичном конусе, то при каждом q отображение гомологий

$$
\left(\alpha_{0}\right)_{*}: H_{q}(C, A) \rightarrow H_{q}(J, A)
$$

равно гомоморфизму

$$
(-\alpha)_{*}: H_{q}(C, A) \rightarrow H_{q}(J, A)
$$

а поэтому мономорфно.

Из этого предложения получаем

СЛЕДСТВИЕ 1.6. Если кривая $C$ лежит на квадратичном конусе, то при каждом q отображение гомологий

$$
\left(\lambda_{0}\right)_{*}: H_{q}(C, A) \rightarrow H_{q}\left(C^{(2)}, A\right)
$$

мономорфно.

1.3. Пространство вещественных канонических $M$-кривых. Далее в этом пункте $(C, \tau)$ - неособая $M$-кривая в проективном пространстве $\mathbb{P}_{Y}^{3}$ с однородными координатами $\left(Y_{1}, Y_{2}, Y_{3}, Y_{4}\right)$, которая является неособым полным пересечением квадрики $Q \subset \mathbb{P}_{Y}^{3}$, заданной уравнением с вешественными коэффищиентами

$$
q(Y) \equiv \sum_{1 \leqslant i, j \leqslant 4} a_{i j} Y_{i} Y_{j}=0
$$


и кубики, заданной уравнением с вещественными коэффициентами

$$
c(Y) \equiv \sum_{1 \leqslant i, j, k \leqslant 4} b_{i j k} Y_{i} Y_{j} Y_{k}=0 .
$$

Вешественная часть $\mathbb{R} C$ кривой $C$ состоит из пяти компонент связности $\Gamma_{0}, \ldots, \Gamma_{4}$, так как $(C, \tau)$ является $M$-кривой, а род кривой $C$ равен 4 . Будем предполагать, что фиксированная точка $c \in C$ принадлежит компоненте $\Gamma_{0}$. Обозначим через $M \mathcal{C}_{Q}$ пространство канонических $M$-кривых на квадрике $Q$. Вещественная часть $\mathbb{R} Q$ квадрики $Q$ может быть эллипсоидом, гиперболоидом или квадратичным конусом. Если $\mathbb{R} Q$ - эллипсоид, то пространство $M \mathcal{C}_{Q}$ состоит из одной компоненты связности, а если $\mathbb{R} Q$ - гиперболоид или квадратичный конус, то пространство $M \mathcal{C}_{Q}$ состоит из двух компонент связности (см. [4], [5]). Через $M \mathcal{C}$ обозначим пространство всех вещественных канонических $M$-кривых. Тогда имеем отображение $\pi: M \mathcal{C} \rightarrow \mathbb{R} \mathcal{Q}$, заданное правилом $\pi(C)=Q(C)$.

Рассмотрим теперь пространство вещественных квадрик $\mathbb{R} \mathcal{Q}$. Оно состоит из квадрик $Q \in \mathcal{Q}$, заданных уравнениями с вещественными коэффициентами $a_{i j}$. Для вешественных квадрик $Q \in \mathbb{R} \mathcal{Q}_{k}$ определена сигнатура $(p, n)$, где $p, n$ - число положительных и отрицательных квадратов в каноническом представлении квадратичной формы

$$
q(Y)=\sum_{i, j=1}^{4} a_{i j} Y_{i} Y_{j} .
$$

Чтобы сигнатура была определена однозначно, будем полагать, что выполняется неравенство $p \geqslant n$, в противном случае форму $q(Y)$ нужно умножить на -1 . Через $\mathcal{Q}_{n}^{p}$ будем обозначать пространство вещественных квадрик $Q \in \mathbb{R} \mathcal{Q}_{k}$ с сигнатурой $(p, n)$, где $k=p+n$. Каждое из открытых множеств $\mathcal{Q}_{n}^{p} \subset \mathbb{R} \mathcal{Q}_{p+n}$ является связным, т. е. является одной из связных компонент в евклидовой топологии квазипроективного многообразия $\mathbb{R} \mathcal{Q}_{p+n}$. Компоненты $\mathcal{Q}_{n}^{p}$ классифищируются по множествам вещественных точек квадрик $Q \in \mathcal{Q}_{n}^{p}$. Если $Q \in \mathcal{Q}_{0}^{4}$, то множество $\mathbb{R} Q$ пустое. Если $Q \in \mathcal{Q}_{1}^{3}$, то множество $\mathbb{R} Q$ - эллипсоид. Если $Q \in \mathcal{Q}_{2}^{2}$, то множество $\mathbb{R} Q$ - гиперболоид. Если $Q \in \mathcal{Q}_{0}^{3}$, то множество $\mathbb{R} Q$ состоит из одной точки. Если $Q \in \mathcal{Q}_{1}^{2}$, то множество $\mathbb{R} Q$ - обычный конус. Если $Q \in \mathcal{Q}_{0}^{2}$, то множество $\mathbb{R} Q$ состоит из одной прямой. Если $Q \in \mathcal{Q}_{1}^{1}$, то множество $\mathbb{R} Q$ состоит из двух плоскостей. Если $Q \in \mathcal{Q}_{0}^{1}$, то множество $\mathbb{R} Q$ состоит из одной плоскости. Заметим еше, что компонента $\mathcal{Q}_{s}^{r}$ лежит в гранище компоненты $\mathcal{Q}_{n}^{p}$, если выполняются следующие условия: $r \leqslant p, s \leqslant n$ и $r+s<p+n$.

Таким образом, отображение $\pi: M \mathcal{C} \rightarrow \pi(M \mathcal{C})$ удовлетворяет следуюшим свойствам: образ $\pi(M \mathcal{C})$ равен связному открытому множеству $\mathcal{Q}_{1}^{3} \cup \mathcal{Q}_{2}^{2} \cup \mathcal{Q}_{1}^{2} \subset \mathbb{R} \mathcal{Q}$; прообраз $\pi^{-1}(Q)$ состоит из одной компоненты связности, если квадрика $Q$ принадлежит открытому множеству $\mathcal{Q}_{1}^{3}$, в противном случае прообраз $\pi^{-1}(Q)$ состоит из двух компонент связности. Через $M \mathcal{C}_{n}^{p}$ будем обозначать пространство вещественных канонических $M$-кривых на квадриках из пространства $\mathcal{Q}_{n}^{p}$. Тогда получим три локально тривиальных расслоения

$$
\pi: M \mathcal{C}_{1}^{3} \rightarrow \mathcal{Q}_{1}^{3}, \quad \pi: M \mathcal{C}_{2}^{2} \rightarrow \mathcal{Q}_{2}^{2}, \quad \pi: M \mathcal{C}_{1}^{2} \rightarrow \mathcal{Q}_{1}^{2}
$$


ПРЕДЛОЖЕНИЕ 1.7. Топологические пространства $\mathrm{MC}_{1}^{3},{M \mathcal{C}_{2}^{2}}^{2}, \mathrm{CC}_{1}^{2}, M \mathcal{C}$ связные.

ДоказАТЕЛьство. Так как локально тривиальное расслоение $\pi: M \mathcal{C}_{1}^{3} \rightarrow \mathcal{Q}_{1}^{3}$ имеет связную базу и связный слой, то пространство расслоения $M \mathcal{C}_{1}^{3}$ тоже связное.

Расслоение $\pi: M \mathcal{C}_{2}^{2} \rightarrow \mathcal{Q}_{2}^{2}$ имеет связную базу, но слой $M \mathcal{C}_{Q}$, где $Q \in \mathcal{Q}_{2}^{2}$, состоит из двух компонент связности. Эти компоненты имеют следующее описание. На гиперболоиде $\mathbb{R} Q$ имеются два семейства прямых. Выберем по представителю $l^{\prime}, l^{\prime \prime}$ из этих семейств и зафиксируем на них ориентацию. Упорядоченный набор ориентированных прямых $l^{\prime}, l^{\prime \prime}$ определяет ориентацию на гиперболоиде. На компонентах $\Gamma_{0}, \ldots, \Gamma_{4}$ кривой $\mathbb{R} C$, где $C \in M \mathcal{C}_{Q}$, также зафиксируем ориентацию. Тогда определены индексы пересечения $\left(\Gamma_{i}, l^{\prime}\right),\left(\Gamma_{i}, l^{\prime \prime}\right)$. Упорядоченная пара этих чисел $\left(\left(\Gamma_{i}, l^{\prime}\right),\left(\Gamma_{i}, l^{\prime \prime}\right)\right)$ составляет бистепень компоненты $\Gamma_{i}$. Если ориентации прямых $l^{\prime}, l^{\prime \prime}$ зафиксированы, то при изменении ориентации компоненты $\Gamma_{i}$ ее бистепень умножается на -1 . Поэтому можем считать, что первое число в бистепени компоненты неотрицательно. Тогда оказывается, что для $M$-кривой справедливо утверждение (см. [5]): одна компонента имеет бистепень $(1, \pm 1)$, а четыре другие компоненты имеют бистепень $(0,0)$, причем компоненты бистепени $(0,0)$ не образуют гнезд, т. е. содержатся вне друг друга. Будем предполагать, что компонента $\Gamma_{0}$ имеет бистепень $(1, \pm 1)$. Тогда компоненты слоя $M \mathcal{C}_{Q}$ характеризуются бистепенью компоненты $\Gamma_{0}$ : функция $C \mapsto\left(\Gamma_{0}, l^{\prime \prime}\right)$ различает компоненты слоя $M \mathcal{C}_{Q}$.

Чтобы доказать связность пространства $M \mathcal{C}_{2}^{2}$, достаточно для фиксированной квадрики $Q \in \mathcal{Q}_{2}^{2}$ и фиксированной канонической кривой $C \in M \mathcal{C}_{Q}$ построить путь $C(t), 0 \leqslant t \leqslant 1$, в пространстве $M \mathcal{C}_{2}^{2}$ такой, что $C(0)=C$, а кривая $C_{1}=C(1)$ принадлежит слою $M \mathcal{C}_{Q}$, но лежит в другой компоненте связности этого слоя. Мы можем считать, что квадрика $Q$ определяется уравнением $Y_{1} Y_{3}+Y_{2} Y_{4}=0$, а прямые $l^{\prime}, l^{\prime \prime}$ на гиперболоиде $\mathbb{R} Q$ задаются системами уравнений

$$
l^{\prime}: Y_{1}=Y_{2}=0, \quad l^{\prime \prime}: Y_{1}=Y_{4}=0 .
$$

Рассмотрим путь $g(t)$ в группе $\mathrm{PGL}_{4} \mathbb{R}$, заданный семейством матриц

$$
G(t)=\left(\begin{array}{cccc}
1 & 0 & 0 & 0 \\
0 & 1 & 0 & 0 \\
0 & 0 & \cos (\pi t) & \sin (\pi t) \\
0 & 0 & -\sin (\pi t) & \cos (\pi t)
\end{array}\right)
$$

Тогда путь $C(t)=g(t)(C)$ в пространстве $M \mathcal{C}_{2}^{2}$ будет искомым путем.

Доказательство связности пространства $M \mathcal{C}_{1}^{2}$ проводится аналогично. В [4] имеется описание компонент слоя $M \mathcal{C}_{Q}$, где $Q \in M \mathcal{C}_{1}^{2}$. На конусе $\mathbb{R} Q$ имеется одно семейство прямых, выберем одну из них, зафиксируем на ней ориентацию и обозначим ее через $l$. Тогда только для одной из компонент $\Gamma_{i}, 0 \leqslant i \leqslant 4$, индекс пересечения $\left(\Gamma_{i}, l\right)$ равен \pm 1 (знак зависит от выбора ориентации компоненты $\Gamma_{i}$ и ориентации конуса), а для остальных компонент он равен нулю. Будем предполагать, что $\left(\Gamma_{0}, l\right)= \pm 1$. Пусть $O \in \mathbb{R} Q$ - вершина конуса, тогда компонента $\Gamma_{0}$ и вершина $O$ разбивают конус на две части, причем оказывается, что все остальные компоненты 
$\Gamma_{1}, \ldots, \Gamma_{4}$ содержатся в одной части конуса. Две компоненты слоя $M \mathcal{C}_{Q}$ характеризуются расположением компонент $\Gamma_{1}, \ldots, \Gamma_{4}$ относительно компоненты $\Gamma_{0}$ : они могут все располагаться до компоненты $\Gamma_{0}$, если двигаться по направлению прямой $l$ от вершины $O$, а могут располагаться после компоненты $\Gamma_{0}$. Чтобы доказать связность пространства $M \mathcal{C}_{1}^{2}$, достаточно для фиксированной квадрики $Q \in \mathcal{Q}_{1}^{2}$ и фиксированной канонической кривой $C \in M \mathcal{C}_{Q}$ построить путь $C(t), 0 \leqslant t \leqslant 1$, в пространстве $M \mathcal{C}_{1}^{2}$ такой, что $C(0)=C$, а кривая $C_{1}=C(1)$ принадлежит слою $M \mathcal{C}_{Q}$, но лежит в другой компоненте связности этого слоя. Мы можем считать, что квадрика $Q$ определяется уравнением $Y_{2}^{2}+Y_{3}^{2}-Y_{4}^{2}=0$. Рассмотрим путь $g(t)$ в группе $\mathrm{PGL}_{4} \mathbb{R}$, заданный семейством матриц $G(t)$. Тогда путь $C(t)=g(t)(C)$ в пространстве $M \mathcal{C}_{1}^{2}$ будет искомым путем.

Для того чтобы установить связность всего пространства канонических $M$-кривых $M \mathcal{C}$, зафиксируем кривую $C_{0} \in M \mathcal{C}_{1}^{2}$ и построим путь $C(t),-\varepsilon \leqslant t \leqslant \varepsilon$, $\varepsilon>0$, в пространстве $M \mathcal{C}$ такой, что $C(0)=C_{0}, C(\varepsilon) \in M \mathcal{C}_{2}^{2}, C(-\varepsilon) \in M \mathcal{C}_{1}^{3}$. Из связности пространств $M \mathcal{C}_{1}^{2}, M \mathcal{C}_{2}^{2}, M \mathcal{C}_{1}^{3}$ следует, что из сушествования такого пути $C(t)$ вытекает связность пространства всех канонических $M$-кривых.

Пусть кривая $C_{0}$ задается системой уравнений

$$
\begin{aligned}
q_{0}(Y) & =\sum_{i, j} a_{i j} Y_{i} Y_{j}=0, \\
c(Y) & =\sum_{i, j, k} b_{i j k} Y_{i} Y_{j} Y_{k}=0 .
\end{aligned}
$$

Пусть $Q_{0}=Q\left(C_{0}\right)$, т.е. квадрика $Q_{0}$ задана уравнением $q_{0}(Y)=0$. Тогда рассмотрим путь $Q(t),-\varepsilon \leqslant t \leqslant \varepsilon$, в пространстве $\mathbb{R} \mathcal{Q}$, проходяший трансверсально к $\mathcal{Q}_{1}^{2}$ через точку $Q_{0}$ при $t=0$, причем $Q(t) \in \mathcal{Q}_{1}^{3}$ при $t<0$ и $Q(t) \in \mathcal{Q}_{2}^{2}$ при $t>0$. Мы можем считать, что семейство квадрик $Q(t)$ задается уравнением

$$
q_{t}(Y)=\sum_{i, j} a_{i j}(t) Y_{i} Y_{j}=0
$$

где коэффициенты $a_{i j}(t)$ - регулярные функции от $t$, причем $a_{i j}(0)=a_{i j}$. Тогда искомый путь $C(t)$ определяется системой уравнений

$$
\begin{aligned}
q_{t}(Y) & =\sum_{i, j} a_{i j}(t) Y_{i} Y_{j}=0, \\
c(Y) & =\sum_{i, j, k} b_{i j k} Y_{i} Y_{j} Y_{k}=0 .
\end{aligned}
$$

Предложение доказано.

1.4. Симметрический квадрат канонической $M$-кривой на гиперболоиде. Пусть $(C, \tau)$ - каноническая кривая из $M \mathcal{C}_{2}^{2}$. Тогда отображения

$$
\lambda^{\prime}:(C, \tau) \rightarrow\left(C^{(2)}, \tau\right), \quad \lambda^{\prime \prime}:(C, \tau) \rightarrow\left(C^{(2)}, \tau\right)
$$

вещественные, т. е. эквивариантные относительно инволюции $\tau$. Действительно, если $C \in M \mathcal{C}_{2}^{2}$ и $Q=Q(C)$, то $\mathbb{R} Q$-гиперболоид. В этом случае каждая комплексная прямая $L$ из какого-либо семейства на квадрике $Q$ под действием комплексного 
сопряжения $\tau: Q \rightarrow Q$ переходит в прямую $\tau(L)$ этого же семейства. Поэтому имеем равенства

$$
\begin{array}{ll}
C \cdot L_{p}^{\prime}=p+p_{1}^{\prime}+p_{2}^{\prime}, & C \cdot L_{\tau(p)}^{\prime}=\tau(p)+\tau\left(p_{1}^{\prime}\right)+\tau\left(p_{2}^{\prime}\right), \\
C \cdot L_{p}^{\prime \prime}=p+p_{1}^{\prime \prime}+p_{2}^{\prime \prime}, & C \cdot L_{\tau(p)}^{\prime \prime}=\tau(p)+\tau\left(p_{1}^{\prime \prime}\right)+\tau\left(p_{2}^{\prime \prime}\right) .
\end{array}
$$

Следовательно,

$$
\lambda^{\prime}(\tau(p))=\tau\left(p_{1}^{\prime}\right)+\tau\left(p_{2}^{\prime}\right)=\tau\left(\lambda^{\prime}(p)\right), \quad \lambda^{\prime \prime}(\tau(p))=\tau\left(p_{1}^{\prime \prime}\right)+\tau\left(p_{2}^{\prime \prime}\right)=\tau\left(\lambda^{\prime \prime}(p)\right) .
$$

ПРЕДЛОЖЕНИЕ 1.8. Отображения (1.3) гомотопны в классе эквивариантных дифференцируемых отображений.

ДоказАтельство. Рассмотрим путь $C(t), 0 \leqslant t \leqslant \varepsilon$, в пространстве $M \mathcal{C}$, заданный системой уравнений (1.2). Гладко продолжим это путь в пространстве $M \mathcal{C}_{2}^{2}$ при $\varepsilon \leqslant t \leqslant 1$, чтобы получить путь, соединяющий точку $C_{0} \in M \mathcal{C}_{1}^{2}$ с точкой $C \in M \mathcal{C}_{2}^{2}$. Далее нужно повторить рассуждения, используемые при доказательстве предложения 1.2. Предложение доказано.

При комплексном сопряжении кривые $C^{\prime}, C^{\prime \prime}$ переходят в себя, т. е. кривые $C^{\prime}, C^{\prime \prime}$ вешественные, а также отображения $\lambda^{\prime}: C \rightarrow C^{\prime}, \lambda^{\prime \prime}: C \rightarrow C^{\prime \prime}$ вещественные. Через $\Gamma_{i}^{\prime}, \Gamma_{i}^{\prime \prime}, i=0, \ldots, 4$, обозначим образы компонент $\Gamma_{i}$ при отображениях $\lambda^{\prime}, \lambda^{\prime \prime}$.

Рассмотрим отображения

$$
\lambda_{\mathbb{R}}^{\prime}: \mathbb{R} C \rightarrow \mathbb{R} C^{(2)}, \quad \lambda_{\mathbb{R}}^{\prime \prime}: \mathbb{R} C \rightarrow \mathbb{R} C^{(2)},
$$

которые являются ограничениями на вешественные точки отображений

$$
\lambda^{\prime}: C \rightarrow C^{(2)}, \quad \lambda^{\prime \prime}: C \rightarrow C^{(2)} .
$$

Из предложения 1.8 следует, что $\lambda_{\mathbb{R}}^{\prime}, \lambda_{\mathbb{R}}^{\prime \prime}$ гомотопны, поэтому каждая компонента связности $\Gamma_{i}, i=0, \ldots, 4$, при отображениях $\lambda_{\mathbb{R}}^{\prime}, \lambda_{\mathbb{R}}^{\prime \prime}$ переходит в одну и ту же компоненту связности множества $\mathbb{R} C^{(2)}$. Следовательно, каждая пара компонент $\Gamma_{i}^{\prime}$, $\Gamma_{i}^{\prime \prime}, i=0, \ldots, 4$, лежит в одной и той же компоненте множества $\mathbb{R} C^{(2)}$. Так как компонента $\Gamma_{0}$ имеет на гиперболоиде $\mathbb{R} Q$ бистепень $(1, \pm 1)$, а компоненты $\Gamma_{1}, \ldots, \Gamma_{4}$ имеют бистепень $(0,0)$, то компоненты $\Gamma_{0}^{\prime}, \Gamma_{0}^{\prime \prime}$ лежат на неориентируемой поверхности $\Pi={ }_{5} S^{2}$, а каждая пара компонент $\Gamma_{i}^{\prime}, \Gamma_{i}^{\prime \prime}, i=1, \ldots, 4$, лежит на торе $T_{0 i}$. Действительно, если $p \in \Gamma_{0}$ и $C \cdot L_{p}^{\prime}=p+p_{1}^{\prime}+p_{2}^{\prime}$, то точки $p_{1}^{\prime}, p_{2}^{\prime}$ либо комплексно-сопряженные, либо принадлежат одной из компонент $\Gamma_{1}, \ldots, \Gamma_{4}$. В любом случае сумма $p_{1}^{\prime}+p_{2}^{\prime}=\lambda^{\prime}(p)$ принадлежит компоненте П. Следовательно, кривая $\Gamma_{0}^{\prime}=\lambda^{\prime}\left(\Gamma_{0}\right)$ лежит на этой компоненте. Аналогично доказывается утверждение для компоненты $\Gamma_{0}^{\prime \prime}$. Если $p \in \Gamma_{1}$ и $C \cdot L_{p}^{\prime}=p+p_{1}^{\prime}+p_{2}^{\prime}$, то одна из точек $p_{1}^{\prime}, p_{2}^{\prime}$ должна принадлежать компоненте $\Gamma_{0}$, а другая - компоненте $\Gamma_{1}$. Поэтому кривая $\Gamma_{1}^{\prime}=\lambda^{\prime}\left(\Gamma_{1}\right)$ лежит на торе $T_{01}$. Аналогично доказывается утверждение для компоненты $\Gamma_{1}^{\prime \prime}$, а также для компонент $\Gamma_{i}^{\prime}, \Gamma_{i}^{\prime \prime}$ при $i>1$.

Поскольку $(C, \tau)$ является $M$-кривой, то компоненты $\Gamma_{0}, \ldots, \Gamma_{4}$ разбивают топологическую поверхность $C$ на две половинки. Выберем одну из этих половинок, 
тогда ее ориентация определит ориентацию сразу всех компонент $\Gamma_{0}, \ldots, \Gamma_{4}$. Поэтому определены классы гомологий

$$
\left[\Gamma_{0}\right], \ldots,\left[\Gamma_{4}\right] \in H_{1}(C, A),
$$

где $A$ - абелева группа. Для них выполнено только одно соотношение

$$
\left[\Gamma_{0}\right]+\cdots+\left[\Gamma_{4}\right]=0
$$

Ориентация компоненты $\Gamma_{i}, i=0, \ldots, 4$, индуцирует ориентацию на компонентах $\Gamma_{i}^{\prime}, \Gamma_{i}^{\prime \prime}$, поэтому определены классы гомологий

$$
\left[\Gamma_{i}^{\prime}\right],\left[\Gamma_{i}^{\prime \prime}\right] \in H_{1}\left(\mathbb{R} C^{(2)}, A\right), \quad i=0, \ldots, 4 .
$$

В силу предложения 1.8 отображения

$$
\lambda_{\mathbb{R}}^{\prime}: \mathbb{R} C \rightarrow \mathbb{R} C^{(2)}, \quad \lambda_{\mathbb{R}}^{\prime \prime}: \mathbb{R} C \rightarrow \mathbb{R} C^{(2)}
$$

гомотопны, поэтому классы гомологий $\left[\Gamma_{i}^{\prime}\right],\left[\Gamma_{i}^{\prime \prime}\right]$ совпадают. Поскольку классы гомологий

$$
\left[\Gamma_{i}\right] \in H_{1}(C, A), \quad i=0, \ldots, 4,
$$

не равны нулю, то из следствия 1.4 вытекает, что также не равны нулю классы гомологий

$$
\left[\Gamma_{i}^{\prime}\right],\left[\Gamma_{i}^{\prime \prime}\right] \in H_{1}\left(\mathbb{R} C^{(2)}, A\right), \quad i=0, \ldots, 4
$$

В итоге доказано

ПРЕДЛОЖЕНИЕ 1.9. Справедливы утверждения:

1) отображения

$$
\lambda_{\mathbb{R}}^{\prime}: \mathbb{R} C \rightarrow \mathbb{R} C^{(2)}, \quad \lambda_{\mathbb{R}}^{\prime \prime}: \mathbb{R} C \rightarrow \mathbb{R} C^{(2)}
$$

гомотопны;

2) отображсния групп гомологий

$$
\left(\lambda_{\mathbb{R}}^{\prime}\right)_{*}: H_{q}(\mathbb{R} C, \mathbb{Z}) \rightarrow H_{q}\left(\mathbb{R} C^{(2)}, A\right), \quad\left(\lambda_{\mathbb{R}}^{\prime \prime}\right)_{*}: H_{q}(\mathbb{R} C, \mathbb{Z}) \rightarrow H_{q}\left(\mathbb{R} C^{(2)}, A\right)
$$

совпадают и мономорфны при каждом $q$.

Топологические кривые $\Gamma_{0}^{\prime}, \Gamma_{0}^{\prime \prime}$ разделяют поверхность $\Pi={ }_{5} S^{2}$ на две части, так как они гомологичны друг другу, но не гомологичны нулю. Обозначим части поверхности ${ }_{5} S^{2}=\Pi$, на которые она разбивается кривыми $\Gamma_{0}^{\prime}, \Gamma_{0}^{\prime \prime}$, через $\Pi^{\prime}, \Pi^{\prime \prime}$. Попробуем выяснить топологический тип этих поверхностей с краем. Так как кривые $\Gamma_{0}^{\prime}, \Gamma_{0}^{\prime \prime}$ гомотопны, то одна из поверхностей $\Pi^{\prime}, \Pi^{\prime \prime}$ должна быть цилиндром, т. е. произведением окружности на отрезок. Будем считать, что поверхность $\Pi^{\prime}$ - цилиндр. Покажем, что поверхность $\Pi^{\prime \prime}$ неориентируемая. Действительно, край поверхности $\Pi^{\prime \prime}$ состоит из двух окружностей, а эйлерова характеристика $\chi\left(\Pi^{\prime \prime}\right)$ равна -3 , поэтому поверхность $\Pi^{\prime \prime}$ неориентируемая. Следовательно, поверхность $\Pi^{\prime \prime}$ является неориентируемой поверхностью рода 3 с двумя дырами. Топологические кривые $\Gamma_{i}^{\prime}, \Gamma_{i}^{\prime \prime}, i=1, \ldots, 4$, разделяют тор $T_{0 i}$ на два цилиндра $\Pi_{i}^{\prime}, \Pi_{i}^{\prime \prime}$, так как кривые $\Gamma_{i}^{\prime}, \Gamma_{i}^{\prime \prime} \subset T_{0 i}$ гомологичны друг другу, но не гомологичны нулю.

Таким образом, справедливо 
ПРЕДЛОЖенИЕ 1.10. Топологические кривые $\Gamma_{i}^{\prime}, \Gamma_{i}^{\prime \prime}, i=1, \ldots, 4$, разделяют тор $T_{0 i}$ на два иилиндра $\Pi_{i}^{\prime}, \Pi_{i}^{\prime \prime}$. Топологические кривые $\Gamma_{0}^{\prime}, \Gamma_{0}^{\prime \prime}$ разделяют поверхность $\Pi={ }_{5} S^{2}$ на две части $\Pi^{\prime}, \Pi^{\prime \prime}$, первая из которых гомеоморфна иилиндру, а вторая является неориентируемой поверхностью рода 3 с двумя дырами.

\section{§2. Кубика с особой двойной точкой и ее поверхность Фано}

Пусть теперь $V_{0}$ - трехмерное особое комплексное алгебраическое многообразие в комплексном проективном пространстве $\mathbb{P}^{4}$, заданное уравнением

$$
P_{0}\left(X_{0}, X_{1}, X_{2}, X_{3}, X_{4}\right)=0,
$$

где $P_{0}(X)=P_{0}\left(X_{0}, X_{1}, X_{2}, X_{3}, X_{4}\right)$ - однородньй многочлен третьей степени с вещественными коэффициентами. Будем предполагать, что точка $p_{0}=[1,0,0,0,0]-$ единственная особая точка многообразия $V_{0}$. В аффинных координатах

$$
Y_{1}=\frac{X_{1}}{X_{0}}, \quad Y_{2}=\frac{X_{2}}{X_{0}}, \quad Y_{3}=\frac{X_{3}}{X_{0}}, \quad Y_{4}=\frac{X_{4}}{X_{0}}
$$

уравнение многообразия $V_{0}$ примет вид

$$
\sum_{1 \leqslant i, j \leqslant 4} a_{i j} Y_{i} Y_{j}+\sum_{1 \leqslant i, j, k \leqslant 4} b_{i j k} Y_{i} Y_{j} Y_{k}=0 .
$$

Мы предполагаем, что особая точка $p_{0}$ является простой двойной точкой. Тогда матрица $\left(a_{i j}\right)$ невырожденная. Можно считать, что гиперплоскость $X_{0}=0$ не касается многообразия $V_{0}$, в противном случае необходимо сделать замену координат, причем такую, что точка $p_{0}$ сохранит координаты $[1,0,0,0,0]$. Тогда пересечение многообразия $V_{0}$ с этой гиперплоскостью является неособой кубической поверхностью $S$. Гиперплоскость $X_{0}=0$ будем обозначать через $Y$. Поверхность $S$ содержится в проективном пространстве $Y$ с однородными координатами $Y_{1}, Y_{2}, Y_{3}, Y_{4}$ и задается однородным уравнением

$$
\sum_{1 \leqslant i, j, k \leqslant 4} b_{i j k} Y_{i} Y_{j} Y_{k}=0 .
$$

Рассмотрим центральную проекцию многообразия $V_{0}$ с центром в точке $p_{0}$ на проективное пространство $Y$. Прямая в $\mathbb{P}^{4}$, проходящая через точку $p_{0}$, либо пересекает многообразие $V_{0}$ еше в одной точке, отличной от $p_{0}$, либо точка $p_{0}$ является тройной общей точкой прямой и многообразия $V_{0}$, либо прямая целиком лежит на многообразии $V_{0}$. Каждая такая прямая пересекает проективное пространство $Y$ в одной точке.

Пусть $C$ - кривая в $Y$, которая является пересечением квадрики $Q \subset Y$, заданной уравнением

$$
\sum_{1 \leqslant i, j \leqslant 4} a_{i j} Y_{i} Y_{j}=0,
$$


и кубики $S$, заданной уравнением

$$
\sum_{1 \leqslant i, j, k \leqslant 4} b_{i j k} Y_{i} Y_{j} Y_{k}=0 .
$$

Тогда кривая $C$ является неособым полным пересечением квадрики и кубики, т. е. $C$-каноническая кривая рода 4 . Это утверждение вытекает из единственности особой точки $p_{0}$ в многообразии $V_{0}$. Каждая прямая в $\mathbb{P}^{4}$, проходящая через точку $p_{0}$ и произвольную точку на кривой $C$, целиком содержится в многообразии $V_{0}$. Также верно обратное утверждение: если прямая, проходящая через точку $p_{0}$, целиком содержится на многообразии $V_{0}$, то она пересекает кривую $C$ в некоторой точке. Каждая прямая в $\mathbb{P}^{4}$, проходящая через точку $p_{0}$ и произвольную точку на $Q \backslash C$, имеет тройную общую точку с многообразием $V_{0}$, а также верно обратное утверждение.

Заметим, что тройка $(Y, Q, C)$ может быть определена инвариантным образом, т. е. без введения координат. В качестве проективного пространства $Y$ необходимо взять множество прямых в $\mathbb{P}^{4}$, проходящих через точку $p_{0}$. Касательный конус к кубике $V_{0}$ дает квадрику $Q \subset Y$, а кривая $C \subset Q$ состоит из прямых на кубике $V_{0}$, проходящих через точку $p_{0}$.

Центральная проекция многообразия $V_{0}$ c центром в точке $p_{0}$ на проективное пространство $Y$ определяет бирациональное отображение $\pi: V_{0} \rightarrow Y$. Отображение проекции $\pi$ не определено в точке $p_{0} \in V_{0}$, а обратное отображение $\pi^{-1}$ не определено в точках из кривой $C \subset Y$. Пусть $\sigma_{p_{0}}: \widetilde{\mathbb{P}}^{4} \rightarrow \mathbb{P}^{4}-$ раздутие с центром в точке $p_{0}$, т. е. $\sigma$-процесс с центром $p_{0}$. Обозначим через $\widetilde{V}_{0}$ собственный прообраз особого многообразия $V_{0}$ при отображении $\sigma_{p_{0}}: \widetilde{\mathbb{P}}^{4} \rightarrow \mathbb{P}^{4}$. Тогда при отображении $\sigma_{p_{0}}: \widetilde{V}_{0} \rightarrow V_{0}$ прообразом точки $p_{0}$ является квадрика. Если теперь мы рассмотрим $\sigma$-процесс $\sigma_{C}: \widetilde{Y} \rightarrow Y$ с центром в кривой $C$, то продолженное отображение $\tilde{\pi}: \widetilde{V}_{0} \rightarrow \widetilde{Y}$ будет изоморфизмом. При раздутии $\sigma_{p_{0}}: \widetilde{V}_{0} \rightarrow V_{0}$ точка $p_{0}$ раздувается в квадрику, а эта квадрика при регулярном отображении $\tilde{\pi}$ отображается на собственный прообраз квадрики $Q$ при раздутии $\sigma_{C}: \widetilde{Y} \rightarrow Y$. Таким образом, если $\widetilde{Q} \subset \widetilde{Y}$ - собственный прообраз квадрики $Q$, то его можно стянуть в простую двойную точку, а полученное многообразие изоморфно многообразию $V_{0}$.

Опишем группы гомологий многообразий, которые появились выше. Род кривой $C$ равен 4. Многообразие $\widetilde{Y}$ получается из проективного пространства $Y$ раздутием кривой $C$, поэтому

$$
H_{1}(\tilde{Y}, \mathbb{Z})=H_{5}(\tilde{Y}, \mathbb{Z})=0, \quad H_{2}(\tilde{Y}, \mathbb{Z})=H_{4}(\tilde{Y}, \mathbb{Z})=\mathbb{Z}^{2}, \quad H_{3}(\tilde{Y}, \mathbb{Z})=\mathbb{Z}^{8} .
$$

Заметим, что если $L_{\widetilde{Y}}^{\prime}, L^{\prime \prime}$ - прямые на квадрике $\widetilde{Q}$ из двух разных семейств прямых, то вложение $\widetilde{Q} \hookrightarrow \widetilde{Y}$ индуцирует точную последовательность

$$
0 \rightarrow \mathbb{Z} \cdot\left[L^{\prime \prime}-L^{\prime}\right] \rightarrow H_{2}(\widetilde{Q}, \mathbb{Z}) \rightarrow H_{2}(\tilde{Y}, \mathbb{Z}) \rightarrow \mathbb{Z} \rightarrow 0 .
$$

Многообразие $V_{0}$ получается из многообразия $\widetilde{Y}$ стягиванием в точку квадрики $\widetilde{Q}$, поэтому при $q>0$ выполняются равенства

$$
H_{q}\left(V_{0}, \mathbb{Z}\right)=H_{q}\left(V_{0},\left\{p_{0}\right\} ; \mathbb{Z}\right)=H_{q}(\widetilde{Y}, \widetilde{Q} ; \mathbb{Z})
$$


Следовательно, из длинной точной последовательности гомологий пары $(\widetilde{Y}, \widetilde{Q})$ вытекают равенства

$$
H_{1}\left(V_{0}, \mathbb{Z}\right)=H_{5}\left(V_{0}, \mathbb{Z}\right)=0, \quad H_{2}\left(V_{0}, \mathbb{Z}\right)=H_{4}\left(V_{0}, \mathbb{Z}\right)=\mathbb{Z}, \quad H_{3}\left(V_{0}, \mathbb{Z}\right)=\mathbb{Z}^{9} .
$$

Поскольку уравнение (2.1) имеет вешественные коэффициенты, то определено множество вещественных решений $\mathbb{R} V_{0}$ этого уравнения. Особая точка $p_{0}$ принадлежит этому множеству, потому что она единственная. После $\sigma$-процесса получаем неособое многообразие $\mathbb{R} \widetilde{V}_{0}$. Оно бирегулярно изоморфно многообразию $\mathbb{R} \tilde{Y}$, где многообразие $\mathbb{R} \widetilde{Y}$ получается из вещественного проективного пространства $\mathbb{R} \mathbb{P}^{3}=\mathbb{R} Y$ с помошью раздутия кривой $\mathbb{R} C$. Если множество $\mathbb{R} Q$ пустое, то множество $\mathbb{R} V_{0}$ состоит из многообразия, диффеоморфного проективному пространству $\mathbb{R} Y$, и изолированной точки $p_{0}$, а многообразия $\mathbb{R} \widetilde{X}_{0}, \mathbb{R} \widetilde{Y}$ состоят из одной компоненты связности, которая диффеоморфна проективному пространству. Если множество $\mathbb{R} Q$ непустое, а множество $\mathbb{R} C$ пустое, то множество $\mathbb{R} Q$ является эллипсоидом, многообразия $\mathbb{R} \widetilde{V}_{0}, \mathbb{R} \widetilde{Y}$ диффеоморфны проективному пространству, а множество $\mathbb{R} V_{0}$ получается стягиванием эллипсоида $\mathbb{R} \widetilde{Q} \subset \mathbb{R} \widetilde{Y}$ в точку. Будем предполагать теперь, что кривая $\mathbb{R} C$ непустая, тогда квадрика $\mathbb{R} Q$ может быть или гиперболоидом, или эллипсоидом.

Заметим, что класс гомологий $[\mathbb{R} \widetilde{Q}] \in H_{2}\left(\mathbb{R} \widetilde{Y}, \mathbb{F}_{2}\right)$ не равен нулю в отличие от класса гомологий $[\mathbb{R} Q] \in H_{2}\left(\underset{\mathbb{R}}{\mathbb{R}}, \mathbb{F}_{2}\right)$. Действительно, полный прообраз квадрики $\mathbb{R} Q$ гомологичен нулю на $\mathbb{R} \widetilde{Y}$, но он состоит из собственного прообраза $\mathbb{R} \widetilde{Q}$ и линейчатой поверхности $\left(\sigma_{C}\right)^{-1}(\mathbb{R} C)$. Таким образом, квадрика $\mathbb{R} \widetilde{Q}$ гомологична линейчатой поверхности $\left(\sigma_{C}\right)^{-1}(\mathbb{R} C)$, которая не гомологична нулю.

Рассмотрим сначала случай, когда квадрика $\mathbb{R} Q$ является гиперболоидом. Вычислим группы гомологий $H_{*}\left(\mathbb{R} V_{0}, \mathbb{F}_{2}\right)$. Так как тело $\mathbb{R} V_{0}$ получается из тела $\mathbb{R} \tilde{Y}$ стягиванием в точку квадрики $\mathbb{R} \widetilde{Q}$, то рассмотрим длинную точную последовательность групп гомологий пары $(\mathbb{R} \widetilde{Y}, \mathbb{R} \widetilde{Q})$

$$
\ldots \rightarrow H_{q+1}\left(\mathbb{R} \tilde{Y}, \mathbb{R} \widetilde{Q} ; \mathbb{F}_{2}\right) \rightarrow H_{q}\left(\mathbb{R} \widetilde{Q}, \mathbb{F}_{2}\right) \rightarrow H_{q}\left(\mathbb{R} \tilde{Y}, \mathbb{F}_{2}\right) \rightarrow H_{q}\left(\mathbb{R} \tilde{Y}, \mathbb{R} \widetilde{Q} ; \mathbb{F}_{2}\right) \rightarrow \ldots
$$

Заметим, что при $q=0,3$ выполняется равенство $H_{q}\left(\mathbb{R} V_{0}, \mathbb{F}_{2}\right)=\mathbb{F}_{2}$. При $q=0$ оно следует из связности тела $\mathbb{R} V_{0}$, а при $q=3$ оно следует из того факта, что квадрика $\mathbb{R} \widetilde{Q}$ не гомологична нулю на $\mathbb{R} \widetilde{Y}$. Поскольку при $q=1,2$ выполняются равенства

$$
H_{q}\left(\mathbb{R} \tilde{Y}, \mathbb{R} \widetilde{Q} ; \mathbb{F}_{2}\right)=H_{q}\left(\mathbb{R} V_{0},\left\{p_{0}\right\} ; \mathbb{F}_{2}\right)=H_{q}\left(\mathbb{R} V_{0}, \mathbb{F}_{2}\right)
$$

то достаточно вычислить группы $H_{q}\left(\mathbb{R} \widetilde{Y}, \mathbb{R} \widetilde{Q} ; \mathbb{F}_{2}\right)$ при $q=1,2$. Группа $H_{1}\left(\mathbb{R} \widetilde{Q}, \mathbb{F}_{2}\right)$ равна $\left(\mathbb{F}_{2}\right)^{2}$ и порождается двумя прямыми $l^{\prime}, l^{\prime \prime}$ на гиперболоиде $\mathbb{R} \widetilde{Q}$. Сумма этих прямых $l^{\prime}+l^{\prime \prime}$ гомологична нулю на теле $\mathbb{R} \widetilde{Y}$, а каждая из них не гомологична нулю. Поэтому из точной последовательности гомологий получаем равенства

$$
\operatorname{dim} H_{1}\left(\mathbb{R} V_{0}, \mathbb{F}_{2}\right)=\operatorname{dim} H_{1}\left(\mathbb{R} \tilde{Y}, \mathbb{R} \widetilde{Q} ; \mathbb{F}_{2}\right)=\operatorname{dim} H_{1}\left(\mathbb{R} \tilde{Y}, \mathbb{F}_{2}\right)-1 .
$$

Квадрика $\mathbb{R} \widetilde{Q}$ не гомологична нулю на теле $\mathbb{R} \widetilde{Y}$, поэтому из точной последовательности получаем

$$
\operatorname{dim} H_{2}\left(\mathbb{R} V_{0}, \mathbb{F}_{2}\right)=\operatorname{dim} H_{2}\left(\mathbb{R} \tilde{Y}, \mathbb{R} \widetilde{Q} ; \mathbb{F}_{2}\right)=\operatorname{dim} H_{2}\left(\mathbb{R} \tilde{Y}, \mathbb{F}_{2}\right)
$$


Таким образом, в случае, когда квадрика $\mathbb{R} \widetilde{Q}$ является гиперболоидом, выполняется равенство

$$
\operatorname{dim} H_{*}\left(\mathbb{R} V_{0}, \mathbb{F}_{2}\right)=\operatorname{dim} H_{*}\left(\mathbb{R} \tilde{Y}, \mathbb{F}_{2}\right)-1 .
$$

Пусть теперь квадрика $\mathbb{R} \widetilde{Q}$ является эллипсоидом. Тогда из точной последовательности вытекают равенства

$$
\operatorname{dim} H_{1}\left(\mathbb{R} V_{0}, \mathbb{F}_{2}\right)=\operatorname{dim} H_{1}\left(\mathbb{R} \tilde{Y}, \mathbb{F}_{2}\right), \quad \operatorname{dim} H_{2}\left(\mathbb{R} V_{0}, \mathbb{F}_{2}\right)=\operatorname{dim} H_{2}\left(\mathbb{R} \tilde{Y}, \mathbb{F}_{2}\right)-1 .
$$

Следовательно, и в этом случае справедливо равенство (2.2).

Покажем, что справедливо

ПРЕДЛОЖЕнИЕ 2.1. Вещественное алгебраическое многообразие $\left(V_{0}, \tau\right)$ тогда и только тогда будет $M$-многообразием, когда вещественная алгебраическая кривая $(C, \tau)$ является $M$-кривой.

ДоКАЗАТЕЛЬСтво. Необходимо проверить, что выполнение равенства

$$
\operatorname{dim} H_{*}\left(\mathbb{R} V_{0}, \mathbb{F}_{2}\right)=\operatorname{dim} H_{*}\left(V_{0}, \mathbb{F}_{2}\right)
$$

равносильно выполнению равенства

$$
\operatorname{dim} H_{*}\left(\mathbb{R} C, \mathbb{F}_{2}\right)=\operatorname{dim} H_{*}\left(C, \mathbb{F}_{2}\right) .
$$

Поскольку $\operatorname{dim} H_{*}\left(V_{0}, \mathbb{F}_{2}\right)=13, \operatorname{dim} H_{*}\left(C, \mathbb{F}_{2}\right)=10$, то нужно показать, что вьполнение равенства

$$
\operatorname{dim} H_{*}\left(\mathbb{R} V_{0}, \mathbb{F}_{2}\right)=13
$$

равносильно вьполнению равенства

$$
\operatorname{dim} H_{*}\left(\mathbb{R} C, \mathbb{F}_{2}\right)=10 .
$$

Из соотношения (2.2) имеем

$$
\operatorname{dim} H_{*}\left(\mathbb{R} V_{0}, \mathbb{F}_{2}\right)=\operatorname{dim} H_{*}\left(\mathbb{R} C, \mathbb{F}_{2}\right)+3,
$$

откуда вытекает утверждение о равносильности равенств. Предложение доказано.

Если кубика $\left(V_{0}, \tau\right)$ является $M$-многообразием, то гомоморфизм

$$
\tau_{*}: H_{q}\left(V_{0}, \mathbb{F}_{2}\right) \rightarrow H_{q}\left(V_{0}, \mathbb{F}_{2}\right)
$$

будет тождественным отображением для каждого $q$. Это общее свойство $M$-многообразий. Для произвольной кубики $\left(V_{0}, \tau\right)$ гомоморфизм $(2.3)$ всегда будет тождественным отображением при $q \neq 3$. Если же он будет тождественным отображением и при $q=3$, то гомоморфизм

$$
\tau_{*}: H_{1}\left(C, \mathbb{F}_{2}\right) \rightarrow H_{1}\left(C, \mathbb{F}_{2}\right)
$$

будет тождественным отображением. Поэтому из предложения 2.1 вытекает 
СЛЕДСТВИЕ 2.2. Если гомоморфизм (2.3) будет тождественным отображением при $q=3$, то особая кубика $\left(V_{0}, \tau\right)$ становится $М$-многообразием.

Заметим, что аналогичное утверждение справедливо и для неособой кубики $(V, \tau)$ (см., например, [7]).

Далее мы опишем поверхность Фано $F_{0}=F_{V_{0}}$, которая параметризует множество прямых на кубике $V_{0}$. Комплексная поверхность Фано описана в [3], мы будем использовать данное описание. Для этого сохраним все обозначения из $\S 1$. Имеется каноническое вложение $i: C \hookrightarrow F_{0}$. По определению, если $p \in C$, то $i(p)=\overline{p_{0} p}$, где $\overline{p_{0} p}$ - прямая в $\mathbb{P}^{4}$, проходящая через точки $p_{0}, p$. Так как прямая $\overline{p_{0} p}$ лежит на многообразии $V_{0}$, то $\overline{p_{0} p} \in F_{0}$. Мы будем отождествлять образ вложения $i$ : $C \hookrightarrow F_{0}$ с кривой $C$. Существует каноническое регулярное отображение $\lambda: C^{(2)} \rightarrow F_{0}$. Оно устроено следуюшим образом. Пусть $p_{1}+p_{2} \in C^{(2)}$. Проведем через точки $p_{1}, p_{2} \in \mathbb{P}^{4}$ прямую $\overline{p_{1} p_{2}}$, причем если точки $p_{1}, p_{2}$ совпадают и равны точке $p$, то прямая $\overline{p_{1} p_{2}}-$ касательная к кривой $C$ в точке $p$. Через точку $p_{0}$ и прямую $\overline{p_{1} p_{2}}$ проведем плоскость $\overline{p_{0} p_{1} p_{2}} \subset \mathbb{P}^{4}$. Эта плоскость пересекает кубику $V_{0}$ по трем прямым, две из них - это прямые $\overline{p_{0} p_{1}}, \overline{p_{0} p_{2}}$ (если $p_{1}=p_{2}=p$, то плоскость $\overline{p_{0} p_{1} p_{2}}$ касается многообразия $V_{0}$ по прямой $\left.\overline{p_{0} p}\right)$, а третью прямую обозначим через $\lambda\left(p_{1}, p_{2}\right)$. Построенное отображение $\lambda: C^{(2)} \rightarrow F_{0}$ обладает следуюшими свойствами:

1) отображение $\lambda: C^{(2)} \backslash \lambda^{-1}(C) \rightarrow F_{0} \backslash C$ является изоморфизмом;

2) прообраз $\lambda^{-1}(C)$ кривой $C$ состоит из двух непересекаюшихся кривых $C^{\prime}, C^{\prime \prime}$, причем отображения $\lambda: C^{\prime} \rightarrow C, \lambda: C^{\prime \prime} \rightarrow C$ являются изоморфизмами;

3 ) кривые $C^{\prime}, C^{\prime \prime}$ совпадают с одноименными кривыми, рассмотренными в $\S 1$, причем обратные отображения к отображениям $\lambda: C^{\prime} \rightarrow C, \lambda: C^{\prime \prime} \rightarrow C$ совпадают с отображениями $\lambda^{\prime}: C \rightarrow C^{\prime}, \lambda^{\prime \prime}: C \rightarrow C^{\prime \prime}$;

$4)$ кривая $C \subset F_{0}$ является обыкновенной двойной кривой и обратное рациональное отображение $\lambda^{-1}: F_{0} \rightarrow C^{(2)}$ - это нормализация поверхности $F_{0}$.


$C^{(2)}$ с помошью отождествления кривых $C^{\prime}, C^{\prime \prime}$, которое производится с помошью отображения

$$
\lambda^{\prime \prime} \circ\left(\lambda^{\prime}\right)^{-1}: C^{\prime} \rightarrow C^{\prime \prime}
$$

Группа гомологий $H_{*}\left(F_{0}, \mathbb{Z}\right)$ свободная (см., например, [9]), и выполняются равенства (см. [3])

$$
H_{0}\left(F_{0}, \mathbb{Z}\right)=H_{4}\left(F_{0}, \mathbb{Z}\right)=\mathbb{Z}, \quad H_{1}\left(F_{0}, \mathbb{Z}\right)=H_{3}\left(F_{0}, \mathbb{Z}\right)=\mathbb{Z}^{9}, \quad H_{2}\left(F_{0}, \mathbb{Z}\right)=\mathbb{Z}^{37}
$$

Теперь мы можем описать, как устроена вещественная часть $\mathbb{R} F_{0}$ поверхности Фано особой кубики. Сначала рассмотрим случай, когда квадрика $\mathbb{R} Q$ является эллипсоидом. В этом случае кривые $C^{\prime}, C^{\prime \prime}$ на симметрическом квадрате $C^{(2)}$ не имеют вещественных точек, причем $\tau\left(C^{\prime}\right)=C^{\prime \prime}$. Поэтому выполняется равенство $\mathbb{R} F_{0}=\mathbb{R} C^{(2)} \sqcup \mathbb{R} C$. В случае, когда квадрика $\mathbb{R} Q$ является гиперболоидом, будем применять предложение 1.10. Тогда после склейки кривых $\Gamma_{i}^{\prime}, \Gamma_{i}^{\prime \prime} \subset T_{0 i}$, $i=1, \ldots, 4$, из них получится двойная кривая $\Gamma_{i} \subset \mathbb{R} C \subset \mathbb{R} F_{0}$, а из двух цилиндров $\Pi_{i}^{\prime}, \Pi_{i}^{\prime \prime} \subset T_{0 i}$ образуются два тора $T_{i}^{\prime}, T_{i}^{\prime \prime}$, пересекающихся по кривой $\Gamma_{i}$. Возникнут именно торы, так как склейка происходит с сохранением ориентаций 
гомологичных кривых $\Gamma_{i}^{\prime}, \Gamma_{i}^{\prime \prime}$. Каждый тор $T_{0 i}, i=1, \ldots, 4$, преобразуется в поверхность $\bar{T}_{0 i}$ с трансверсальным самопересечением по кривой $\Gamma_{i}$. После склейки кривых $\Gamma_{0}^{\prime}, \Gamma_{0}^{\prime \prime} \subset \Pi$ из них получается двойная кривая $\Gamma_{0} \subset \mathbb{R} C \subset \mathbb{R} F_{0}$, а из поверхностей $\Pi^{\prime}, \Pi^{\prime \prime} \subset \Pi$ - две поверхности $\Pi_{1}, \Pi_{2}$, пересекающиеся по кривой $\Gamma_{0}$. Поверхность $\Pi_{1}$ образуется из цилиндра $\Pi^{\prime}$ склейкой его двух краев $\Gamma_{0}^{\prime}, \Gamma_{0}^{\prime \prime}$ согласно их ориентации, поэтому поверхность $\Pi_{1}$ будет тором. Поверхность $\Pi_{2}$ образуется из неориентируемой поверхности $\Pi^{\prime \prime}$ склейкой ее двух краев $\Gamma_{0}^{\prime}, \Gamma_{0}^{\prime \prime}$ также согласно их ориентации. Поскольку поверхность $\Pi^{\prime \prime}$ имеет род 3 , то поверхность $\Pi_{2}$ гомеоморфна ${ }_{5} S^{2}$. Из всей поверхности П после склейки получится поверхность $\bar{\Pi}$ с трансверсальным самопересечением по кривой $\Gamma_{0}$. Таким образом, доказано

ПРЕДЛОЖенИЕ 2.3. Пусть $(C, \tau)-M$-кривая; тогда справедливы утверждения:

1) если квадрика $\mathbb{R} Q$ является эллипсоидом, то вещественная часть $\mathbb{R} F_{0}$ поверхности Фано $F_{0}$ гомеоморфна несвязной сумме $\mathbb{R} C^{(2)} \sqcup \mathbb{R} C$;

2) если квадрика $\mathbb{R} Q$ является гиперболоидом, то поверхность $\mathbb{R} F_{0}$ получается из поверхности $\mathbb{R} C^{(2)}$ склейкой кривых $\mathbb{R} C^{\prime}, \mathbb{R} C^{\prime \prime}$ с помощью отображения

$$
\lambda_{\mathbb{R}}^{\prime \prime} \circ\left(\lambda_{\mathbb{R}}^{\prime}\right)^{-1}: \mathbb{R} C^{\prime} \rightarrow \mathbb{R} C^{\prime \prime} .
$$

После склейки кривых $\Gamma_{i}^{\prime}, \Gamma_{i}^{\prime \prime} \subset T_{0 i}, i=1, \ldots, 4$, из них получается двойная кривая $\Gamma_{i} \subset \mathbb{R} C \subset \mathbb{R} F_{0}$, а из тора $T_{0 i}$ получается поверхность $\bar{T}_{0 i}$ с трансверсальным самопересечением по кривой $\Gamma_{i}$, причем поверхность $\bar{T}_{0 i}$ состоит из двух топологических торов $T_{i}^{\prime}, T_{i}^{\prime \prime}$, пересекающихся по $\Gamma_{i}$. После склейки кривых $\Gamma_{0}^{\prime}, \Gamma_{0}^{\prime \prime} \subset \Pi$ из них получается двойная кривая $\Gamma_{0} \subset \mathbb{R} C \subset \mathbb{R} F_{0}$, а из поверхности П - поверхность $\bar{\Pi}$ с трансверсальным самопересечением по кривой $\Gamma_{0}$, причем поверхность $\bar{\Pi}$ состоит из двух топологических поверхностей $\Pi_{1}, \Pi_{2}$, пересекающихся по кривой $\Gamma_{0}$, әде поверхность $\Pi_{1}$ гомеоморфна тору $T^{2}$, а поверхность $\Pi_{2}$ гомеоморфна неориентируемой поверхности ${ }_{5} S^{2}$.

Покажем, что справедливо

ПРЕДЛОЖЕНИЕ 2.4. Если кривая $(C, \tau)$ является $M$-кривой, то поверхность Фано $\left(F_{0}, \tau\right)$ будет $M$-поверхностью.

ДокАЗАТЕльство. Сначала покажем, что если квадрика $\mathbb{R} Q$ является эллипсоидом, то предложение справедливо. В этом случае выполняется равенство $\mathbb{R} F_{0}=\mathbb{R} C^{(2)} \sqcup \mathbb{R} C$. Следовательно, имеем равенство для групп гомологий

$$
\operatorname{dim} H_{*}\left(\mathbb{R} F_{0}, \mathbb{F}_{2}\right)=\operatorname{dim} H_{*}\left(\mathbb{R} C^{(2)}, \mathbb{F}_{2}\right)+\operatorname{dim} H_{*}\left(\mathbb{R} C, \mathbb{F}_{2}\right),
$$

T.e.

$$
\operatorname{dim} H_{*}\left(\mathbb{R} F_{0}, \mathbb{F}_{2}\right)=2 k^{2}+7,
$$

где $k$ - число компонент связности множества $\mathbb{R} C$. C другой стороны, имеем равенство

$$
\operatorname{dim} H_{*}\left(F_{0}, \mathbb{F}_{2}\right)=57
$$

Таким образом, равенство

$$
\operatorname{dim} H_{*}\left(\mathbb{R} F_{0}, \mathbb{F}_{2}\right)=\operatorname{dim} H_{*}\left(F_{0}, \mathbb{F}_{2}\right)
$$


будет выполняться тогда и только тогда, когда $k=5$, т.е. когда кривая $(C, \tau)$ является $M$-кривой. Перейдем к случаю, когда квадрика $\mathbb{R} Q$ - гиперболоид. Заметим прежде всего, что достаточно доказать равенство (2.4). Существует длинная точная последовательность гомологий, ассоциированная с отображениeм $\lambda: \mathbb{R} C^{(2)} \rightarrow \mathbb{R} F_{0}:$

$$
\ldots \rightarrow H_{q}\left(\mathbb{R} C, \mathbb{F}_{2}\right) \stackrel{\Lambda_{*}}{\longrightarrow} H_{q}\left(\mathbb{R} C^{(2)}, \mathbb{F}_{2}\right) \stackrel{\lambda_{*}}{\longrightarrow} H_{q}\left(\mathbb{R} F_{0}, \mathbb{F}_{2}\right) \rightarrow H_{q-1}\left(\mathbb{R} C, \mathbb{F}_{2}\right) \stackrel{\Lambda_{*}}{\longrightarrow} \ldots
$$

где гомоморфизм $\Lambda_{*}$ равен разности $\left(\lambda_{\mathbb{R}}^{\prime}\right)_{*}-\left(\lambda_{\mathbb{R}}^{\prime \prime}\right)_{*}$. Эта точна последовательность получается из длинной точной последовательности гомологий пары $\left(W, \mathbb{R} C^{(2)}\right)$, где $W$ - пространство, полученное из $\mathbb{R} C^{(2)}$ приклеиванием к нему цилиндра $\mathbb{R} C \times$ $[0,1]$ : нижнее основание $\mathbb{R} C \times 0$ приклеивается отображением $\lambda_{\mathbb{R}}^{\prime}: \mathbb{R} C \rightarrow \mathbb{R} C^{(2)}$, а верхнее основание $\mathbb{R} C \times 1$ - отображением $\lambda_{\mathbb{R}}^{\prime \prime}: \mathbb{R} C \rightarrow \mathbb{R} C^{(2)}$ (см. построение аналогичной точной последовательности для отображения $\lambda: C^{(2)} \rightarrow F_{0}$ в [3]). Но в силу предложения 1.9 гомоморфизмы

$$
\left(\lambda_{\mathbb{R}}^{\prime}\right)_{*},\left(\lambda_{\mathbb{R}}^{\prime \prime}\right)_{*}: H_{q}\left(\mathbb{R} C, \mathbb{F}_{2}\right) \rightarrow H_{q}\left(\mathbb{R} C^{(2)}, \mathbb{F}_{2}\right)
$$

совпадают при любом $q$. Тогда получим, что гомоморфизмы $\Lambda_{*}$ в точной последовательности (2.5) равны нулю, поэтому из этой последовательности будет вытекать равенство (2.4). Следовательно, предложение доказано.

\section{§3. Неособая кубика и ее поверхность Фано}

Сначала мы изложим идеи доказательства теоремы 0.1. При этом будем использовать эквивариантные когомологии Гротендика $H^{q}\left(F, G ; \mathbb{F}_{2}\right)$, где $G=$ $\{1, \tau\}$ - группа второго порядка. Для них существуют две спектральные последовательности, вторая из которых имеет вид (см. [6])

$$
\mathrm{II}_{2}^{p, q}=H^{p}\left(G, H^{q}\left(F, \mathbb{F}_{2}\right)\right) \Rightarrow H^{p+q}\left(F, G ; \mathbb{F}_{2}\right) .
$$

Если эта спектральная последовательность вырождается и инволюция

$$
\tau^{*}: H^{*}\left(F, \mathbb{F}_{2}\right) \rightarrow H^{*}\left(F, \mathbb{F}_{2}\right)
$$

тривиальна, то поверхность Фано $(F, \tau)$ является $M$-поверхностью (см., например, [7]). Поскольку множество $\mathbb{R} F$ непустое, то с помошью $\cup$-умножения и двойственности Пуанкаре устанавливается, что для вырождения второй спектральной последовательности поверхности достаточно обращения в нуль дифференциалов (см., например, [8])

$$
d_{2}^{0,3}: \mathrm{II}_{2}^{0,3} \rightarrow \mathrm{II}_{2}^{2,2}, \quad d_{3}^{0,3}: \mathrm{II}_{3}^{0,3} \rightarrow \mathrm{II}_{3}^{3,1}
$$

Мы будем использовать несколько утверждений о кольце когомологий поверхности Фано, которые фактически доказаны в [3]. Первое утверждение из [3] формулируется следующим образом.

Лемма 3.1. Существует канонический изоморфизм пространств когомологий

$$
H^{1}\left(F, \mathbb{F}_{2}\right) \stackrel{\approx}{\longrightarrow} H^{3}\left(V, \mathbb{F}_{2}\right),
$$

сохраняющий инволющию $\tau^{*}$.

Второе утверждение из [3] формулируется следующим образом. 
Лемма 3.2. Размерность ядра гомоморфизма $\mathbb{F}_{2}$-пространств когомологий

$$
h: \Lambda^{2} H^{1}\left(F, \mathbb{F}_{2}\right) \rightarrow H^{2}\left(F, \mathbb{F}_{2}\right),
$$

заданного $\cup$-произведением, равна 1 , причем имеет место разложение

$$
H^{2}\left(F, \mathbb{F}_{2}\right)=h\left(\Lambda^{2} H^{1}\left(F, \mathbb{F}_{2}\right)\right) \oplus \mathbb{F}_{2} \cdot[K]^{*},
$$

әде $K$ - канонический дивизор.

Третье утверждение из [3] формулируется следующим образом.

ЛЕмма 3.3. Гомоморфизм пространств когомологий

$$
\Lambda^{3} H^{1}\left(F, \mathbb{F}_{2}\right) \rightarrow H^{3}\left(F, \mathbb{F}_{2}\right),
$$

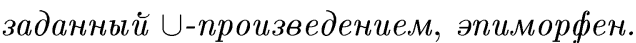

Кубика $(V, \tau)$ будет $M$-многообразием тогда и только тогда, когда инволюция

$$
\tau^{*}: H^{3}\left(V, \mathbb{F}_{2}\right) \rightarrow H^{3}\left(V, \mathbb{F}_{2}\right)
$$

тривиальна (см., например, [7]). Поэтому из леммы 3.1 вытекает, что для доказательства теоремы 0.1 достаточно установить следующее утверждение: если инволюция

$$
\tau^{*}: H^{1}\left(F, \mathbb{F}_{2}\right) \rightarrow H^{1}\left(F, \mathbb{F}_{2}\right)
$$

тривиальна, то поверхность Фано является $M$-поверхностью. Для доказательства этого утверждения нужно применить леммы $3.2,3.3$. Из них вытекает, что инволюция

$$
\tau^{*}: H^{*}\left(F, \mathbb{F}_{2}\right) \rightarrow H^{*}\left(F, \mathbb{F}_{2}\right)
$$

тривиальна, а из леммы 3.3 следует, что дифференциалы

$$
d_{2}^{0,3}: \mathrm{II}_{2}^{0,3} \rightarrow \mathrm{II}_{2}^{2,2}, \quad d_{3}^{0,3}: \mathrm{II}_{3}^{0,3} \rightarrow \mathrm{II}_{3}^{3,1}
$$

равны нулю, так как равны нулю дифференциалы

$$
d_{2}^{0,1}: \mathrm{II}_{2}^{0,1} \rightarrow \mathrm{II}_{2}^{2,0}, \quad d_{3}^{0,1}: \mathrm{II}_{3}^{0,1} \rightarrow \mathrm{II}_{3}^{3,-1} .
$$

На этом мы заканчиваем набросок доказательства теоремы 0.1 , подробное доказательство изложено в [9]. Перейдем к доказательству теоремы 0.2 .

Данная неособая кубика $V$ включается в линейное семейство кубик $V_{t}, t \in \mathbb{P}^{1}$, которое представляет собой пучок гиперплоских сечений неособой четырехмерной кубики $Z \subset \mathbb{P}^{5}$, причем особые сечения содержат только одну простую двойную точку, а других особых точек нет. Это семейство образовано сечениями гиперплоскостей, проходяших через фиксированное трехмерное проективное пространство $R \subset \mathbb{P}^{5}$. Если исходная кубика $V$ вещественная, то существует вещественное линейное семейство $V_{t}$, содержащее данную кубику. Отсюда следует, что оно определено над полем вещественных чисел, а поэтому имеется отображение комплексного сопряжения $\tau: V_{t} \rightarrow V_{\tau(t)}$. В частности, если точка $t$ вешественная, т. е. $t \in \mathbb{R P}^{1}$, то кубика $V_{t}$ вещественная, а также справедливо обратное утверждение. Мы можем считать, что при $t=1$ получаем исходную неособую кубику $V$, 
а ближайшая вешественная особая кубика получается при $t=0$, где $t$ - аффинная координата на $\mathbb{P}^{1}$, в противном случае необходимо сделать соответствующую замену координаты $t$.

ПРЕДЛОЖЕНИЕ 3.4. Если неособая кубика $(V, \tau)$ является $M$-многообразием, то особая кубика $\left(V_{0}, \tau\right)$ будет также $M$-многообразием.

ДОКАЗАТЕЛЬСТВО. Поскольку кубика $(V, \tau)$ является $M$-многообразием, то гомоморфизм $\tau_{*}: H_{3}\left(V, \mathbb{F}_{2}\right) \rightarrow H_{3}\left(V, \mathbb{F}_{2}\right)$ будет тождественным отображением, а нам достаточно показать, что и гомоморфизм $\tau_{*}: H_{3}\left(V_{0}, \mathbb{F}_{2}\right) \rightarrow H_{3}\left(V_{0}, \mathbb{F}_{2}\right)$ является тождественным отображением. Это вытекает из следствия 2.2. Сушествует непрерывное отображение $\varrho: V \rightarrow V_{0}$, удовлетворяющее свойствам (см., например, [11], [1]):

1) прообраз особой точки $p_{0}$ гомеоморфен трехмерной сфере $\Sigma$;

2) отображение $\varrho: V \backslash \Sigma \rightarrow V_{0} \backslash\left\{p_{0}\right\}$ является гомеоморфизмом;

3) отображение $\varrho: V \rightarrow V_{0}$ эквивариантное, т.е. коммутирует с комплексным сопряжением.

Тогда имеет место короткая точная последовательность

$$
0 \rightarrow H_{3}\left(\Sigma, \mathbb{F}_{2}\right) \rightarrow H_{3}\left(V, \mathbb{F}_{2}\right) \stackrel{\varrho_{*}}{\longrightarrow} H_{3}\left(V_{0}, \mathbb{F}_{2}\right) \rightarrow 0
$$

Из этой точной последовательности вытекает нужное нам утверждение. Предложение доказано.

Сферу $\Sigma \subset V$ будем называть исчезающей сферой. Она инвариантна относительно инволюции $\tau$. Выберем такое малое число $0<\varepsilon<1$, чтобы многообразия $V_{t}$ были неособые при $0<|t|<2 \varepsilon$. Заметим, что многообразия $\left(V_{1}, \tau\right),\left(V_{\varepsilon}, \tau\right)$ эквивариантно диффеоморфны. Обозначим кубики $V_{ \pm \varepsilon}$ кратко через $V_{ \pm}$, а исчезаюшие сферы на них - через $\Sigma_{ \pm}$. Эти сферы можно задать в специальных координатах уравнениями. Пусть $\sigma_{R}: \widetilde{\mathbb{P}}^{5} \rightarrow \mathbb{P}^{5}-\sigma$-процесс с центром в пространстве $R$, а $\widetilde{Z}$ - собственный прообраз четырехмерной кубики $Z$ при этом преобразовании. Тогда четырехмерное многообразие $\widetilde{Z}$ образует тотальное многообразие семейства $V_{t}$. В окрестности $W$ точки $p_{0} \in \widetilde{Z}$ существуют голоморфные координаты $w_{1}, w_{2}, w_{3}, w_{4}$ такие, что пересечение $V_{t} \cap W$ задается уравнением

$$
\pm w_{1}^{2} \pm w_{2}^{2} \pm w_{3}^{2} \pm w_{4}^{2}=t
$$

где знаки \pm определяются сигнатурой квадратичной формы

$$
\sum_{1 \leqslant k, l \leqslant 4} a_{k l} y_{k} y_{l},
$$

причем координаты

$$
w_{1}=u_{1}+i v_{1}, \quad w_{2}=u_{2}+i v_{2}, \quad w_{3}=u_{3}+i v_{3}, \quad w_{4}=u_{4}+i v_{4}
$$

являются вешественными в обобшенном смысле, т. е. удовлетворяют условию

$$
w_{k}(\tau(p))=\overline{w_{k}(p)}, \quad k=1, \ldots, 4,
$$


где черта означает комплексное сопряжение. Таким образом, можно считать, что пересечение $V_{t} \cap W$ задается одним из следуюших уравнений:

$$
\begin{array}{r}
w_{1}^{2}+w_{2}^{2}+w_{3}^{2}+w_{4}^{2}=t, \\
w_{1}^{2}+w_{2}^{2}+w_{3}^{2}-w_{4}^{2}=t, \\
w_{1}^{2}+w_{2}^{2}-w_{3}^{2}-w_{4}^{2}=t, \\
w_{1}^{2}-w_{2}^{2}-w_{3}^{2}-w_{4}^{2}=t, \\
-w_{1}^{2}-w_{2}^{2}-w_{3}^{2}-w_{4}^{2}=t,
\end{array}
$$

где нумерация производится с помощью значения сигнатуры, которая состоит из двух чисел, первое означает число плюсов, а второе - минусов. В каждом из этих случаев исчезаюшая сфера $\Sigma_{+}$на $V_{+}=V_{\varepsilon}$ при достаточно малом $\varepsilon>0$ определяется системой уравнений

$$
\begin{array}{lll}
(4,0)_{+} & u_{1}^{2}+u_{2}^{2}+u_{3}^{2}+u_{4}^{2}=\varepsilon, & v_{1}=v_{2}=v_{3}=v_{4}=0, \\
(3,1)_{+} & u_{1}^{2}+u_{2}^{2}+u_{3}^{2}+v_{4}^{2}=\varepsilon, & v_{1}=v_{2}=v_{3}=u_{4}=0, \\
(2,2)_{+} & u_{1}^{2}+u_{2}^{2}+v_{3}^{2}+v_{4}^{2}=\varepsilon, & v_{1}=v_{2}=u_{3}=u_{4}=0, \\
(1,3)_{+} & u_{1}^{2}+v_{2}^{2}+v_{3}^{2}+v_{4}^{2}=\varepsilon, & v_{1}=u_{2}=u_{3}=u_{4}=0, \\
(0,4)_{+} & v_{1}^{2}+v_{2}^{2}+v_{3}^{2}+v_{4}^{2}=\varepsilon, & u_{1}=u_{2}=u_{3}=u_{4}=0 .
\end{array}
$$

В этих случаях множество вещественных точек $\Sigma_{+}^{\tau}$ может быть: трехмерной сферой; двумерной сферой; одномерной сферой, т. е. окружностью; нульмерной сферой, т. е. состоять из двух точек; пустым множеством. Соответственно, исчезающая сфера $\Sigma_{-}$на $V_{-}=V_{-\varepsilon}$ при достаточно малом $\varepsilon>0$ определяется системой уравнений

$$
\begin{array}{lll}
(4,0)_{-} & v_{1}^{2}+v_{2}^{2}+v_{3}^{2}+v_{4}^{2}=\varepsilon, & u_{1}=u_{2}=u_{3}=u_{4}=0, \\
(3,1)_{-} & v_{1}^{2}+v_{2}^{2}+v_{3}^{2}+u_{4}^{2}=\varepsilon, & u_{1}=u_{2}=u_{3}=v_{4}=0, \\
(2,2)_{-} & v_{1}^{2}+v_{2}^{2}+u_{3}^{2}+u_{4}^{2}=\varepsilon, & u_{1}=u_{2}=v_{3}=v_{4}=0, \\
(1,3)_{-} & v_{1}^{2}+u_{2}^{2}+u_{3}^{2}+u_{4}^{2}=\varepsilon, & u_{1}=v_{2}=v_{3}=v_{4}=0, \\
(0,4)_{-} & u_{1}^{2}+u_{2}^{2}+u_{3}^{2}+u_{4}^{2}=\varepsilon, & v_{1}=v_{2}=v_{3}=v_{4}=0 .
\end{array}
$$

В этих случаях множество вещественных точек $\Sigma_{-}^{\tau}$ может быть: пустым множеством; нульмерной сферой, т.е. состоять из двух точек; одномерной сферой, т.е. окружностью; двумерной сферой; трехмерной сферой.

Если особая точка $p_{0} \in V_{0}$ является изолированной точкой множества вещественных точек $\mathbb{R} V_{0}$, то вещественная исчезающая сфера $\Sigma_{ \pm}^{\tau}$ представляет собой или пустое множество, или совпадает со всей сферой $\Sigma_{ \pm}$. Заметим, что если $\left(V_{0}, \tau\right)$ является $M$-многообразием, то точка $p_{0}$ не является изолированной точкой множества вешественных точек $\mathbb{R} V_{0}$, в этом случае вещественная исчезаюшая сфера $\Sigma_{ \pm}^{\tau}$ представляет собой или две точки, или окружность, или двумерную сферу.

Оказывается, что справедливо 
ПРЕДЛОЖЕНИЕ 3.5. Если особая кубика $\left(V_{0}, \tau\right)$ является $M$-многообразием, то одна из неособых кубик $\left(V_{ \pm}, \tau\right)$ будет также $M$-многообразием, причем другая неособая кубика уже не может быть $M$-многообразием.

ДокАЗАТЕльство. Достаточно рассмотреть два случая, когда вешественная исчезаюшая сфера $\Sigma_{+}^{\tau}$ является двумерной сферой или окружностью. Сначала докажем предложение в случае, когда вещественная исчезающая сфера $\Sigma_{+}^{\tau}$ является двумерной сферой. Заметим, что тогда квадрика $\mathbb{R} Q$ - эллипсоид. Покажем, что в этом случае многообразие $\left(V_{+}, \tau\right)$ является $M$-многообразием, а многообразие $\left(V_{-}, \tau\right)$ не является. Для доказательства первого утверждения рассмотрим спектральную последовательность Лере отображения $\varrho: \mathbb{R} V_{+} \rightarrow \mathbb{R} V_{0}$ :

$$
E_{2}^{p, q}=H^{p}\left(\mathbb{R} V_{0}, R^{q} \varrho_{*}\left(\mathbb{F}_{2}\right)\right) \Rightarrow H^{p+q}\left(\mathbb{R} V_{+}, \mathbb{F}_{2}\right) .
$$

Поскольку

$$
R^{q} \varrho_{*}\left(\mathbb{F}_{2}\right)=\left\{\begin{array}{lll}
\mathbb{F}_{2} & \text { при } & q=0, \\
\left.\left(\mathbb{F}_{2}\right)\right|_{p_{0}} & \text { при } & q=2, \\
0 & \text { при } & q \neq 0,2,
\end{array}\right.
$$

то выполняются равенства

$$
E_{2}^{p, q}= \begin{cases}H^{p}\left(\mathbb{R} V_{0}, \mathbb{F}_{2}\right) & \text { при } q=0, \\ \mathbb{F}_{2} & \text { при } p=0, q=2, \\ 0 & \text { при остальных } p, q .\end{cases}
$$

Поверхность $\mathbb{R} \widetilde{Q}$ не гомологична нулю на теле $\mathbb{R} \widetilde{Y}$, поэтому выполняются равенства

$$
H^{3}\left(\mathbb{R} V_{0}, \mathbb{F}_{2}\right)=H^{3}\left(\mathbb{R} \tilde{Y}, \mathbb{R} \widetilde{Q} ; \mathbb{F}_{2}\right)=\mathbb{F}_{2} .
$$

Следовательно, дифференщиал $d_{3}^{0,2}$ равен нулю, а поэтому

$$
\operatorname{dim} H^{*}\left(\mathbb{R} V_{+}, \mathbb{F}_{2}\right)=\operatorname{dim} H^{*}\left(\mathbb{R} V_{0}, \mathbb{F}_{2}\right)+1 .
$$

Тогда из равенств

$$
\operatorname{dim} H^{*}\left(\mathbb{R} V_{0}, \mathbb{F}_{2}\right)=\operatorname{dim} H^{*}\left(V_{0}, \mathbb{F}_{2}\right), \quad \operatorname{dim} H^{*}\left(V_{+}, \mathbb{F}_{2}\right)=\operatorname{dim} H^{*}\left(V_{0}, \mathbb{F}_{2}\right)+1
$$

вытекает выполнение требуемого равенства

$$
\operatorname{dim} H^{*}\left(\mathbb{R} V_{+}, \mathbb{F}_{2}\right)=\operatorname{dim} H^{*}\left(V_{+}, \mathbb{F}_{2}\right) .
$$

Таким образом, в случае, когда множество вешественных точек $\Sigma_{+}^{\tau}$ является сферой, кубика $\left(V_{+}, \tau\right)$ является $M$-многообразием. Покажем, что в этом случае кубика $\left(V_{-}, \tau\right)$ уже не может быть $M$-многообразием. Аналогично рассмотрим спектральную последовательность Лере для отображения $\varrho: \mathbb{R} V_{-} \rightarrow \mathbb{R} V_{0}$. Тогда имеем следуюшие равенства: $R^{q} \varrho_{*}\left(\mathbb{F}_{2}\right)=0$ при $q>0, R^{0} \varrho_{*}\left(\mathbb{F}_{2}\right)=\mathcal{F}$, где для пучка $\mathcal{F}$ на $\mathbb{R} V_{0}$ имеет место точная последовательность пучков

$$
\left.0 \rightarrow \mathbb{F}_{2} \rightarrow \mathcal{F} \rightarrow \mathbb{F}_{2}\right|_{p_{0}} \rightarrow 0
$$


Поэтому имеем равенства для размерностей пространств когомологий:

$$
\begin{aligned}
\operatorname{dim} H^{2}\left(\mathbb{R} V_{-}, \mathbb{F}_{2}\right) & =\operatorname{dim} H^{2}\left(\mathbb{R} V_{0}, \mathcal{F}\right) \\
& =\operatorname{dim} H^{2}\left(\mathbb{R} V_{0}, \mathbb{F}_{2}\right)=\operatorname{dim} H^{2}\left(\mathbb{R} \tilde{Y}, \mathbb{F}_{2}\right)-1=5 .
\end{aligned}
$$

Следовательно,

$$
\operatorname{dim} H^{*}\left(\mathbb{R} V_{-}, \mathbb{F}_{2}\right)=12<14=\operatorname{dim} H^{*}\left(V_{-}, \mathbb{F}_{2}\right)
$$

Переходим к случаю, когда вещественная исчезающая сфера $\Sigma_{+}^{\tau}$ является окружностью. Заметим, что тогда $\Sigma_{-}^{\tau}$ - тоже окружность и рассуждения, которые применялись в предыдущем случае, уже не достаточны. Отметим также, что в этом случае множество вешественных точек $\mathbb{R} Q$ является гиперболоидом. Прежде всего опишем перестройку, с помощью которой из особого многообразия $\mathbb{R} V_{0}$ получаются неособые многообразия $\mathbb{R} V_{ \pm}$(см., например, [10]). Удалим из особого многообразия $\mathbb{R} V_{0}$ точки, удовлетворяющие неравенству

$$
u_{1}^{2}+u_{2}^{2}+u_{3}^{2}+u_{4}^{2}+v_{1}^{2}+v_{2}^{2}+v_{3}^{2}+v_{4}^{2}<2 r^{2}
$$

где $r$ - малое положительное число, причем $\varepsilon \ll r$. Полученное многообразие с краем обозначим через $M$. Так как в окрестности точки $p_{0} \in \mathbb{R} V_{0}$ особое многообразие $\mathbb{R} V_{0}$ задается в координатах $u_{1}, u_{2}, u_{3}, u_{4}$ с помошњю уравнения $u_{1}^{2}+u_{2}^{2}-u_{3}^{2}-u_{4}^{2}=0$, то край многообразия $M$ является тором $T$, заданньм системой уравнений

$$
\begin{aligned}
& u_{1}^{2}+u_{2}^{2}=r^{2} \\
& u_{3}^{2}+u_{4}^{2}=r^{2} .
\end{aligned}
$$

Обозначим через $T_{ \pm}$полнотории в пространстве $\mathbb{R}^{4}$ с координатами $u_{1}, u_{2}$, $u_{3}, u_{4}$, заданные соответственно системами

$$
\begin{aligned}
& T_{+}:\left\{\begin{array}{l}
u_{1}^{2}+u_{2}^{2}=r^{2} \\
u_{3}^{2}+u_{4}^{2} \leqslant r^{2},
\end{array}\right. \\
& T_{-}:\left\{\begin{array}{l}
u_{1}^{2}+u_{2}^{2} \leqslant r^{2} \\
u_{3}^{2}+u_{4}^{2}=r^{2}
\end{array}\right.
\end{aligned}
$$

Тогда многообразия $\mathbb{R} V_{ \pm}$гомеоморфны топологическим многообразиям, которые получаются из многообразия с краем $M$ с помощью объединения соответственно с полноторием $T_{ \pm}$. Полученные из $M$ с помощью объединения с полноториями топологические многообразия будем обозначать через $M_{ \pm}$. Заметим, что они получаются из особого многообразия $\mathbb{R} V_{0}$ заменой конуса

$$
K:\left\{\begin{array}{l}
u_{1}^{2}+u_{2}^{2}+u_{3}^{2}+u_{4}^{2} \leqslant 2 r^{2} \\
u_{1}^{2}+u_{2}^{2}-u_{3}^{2}-u_{4}^{2}=0
\end{array}\right.
$$


полноториями $T_{ \pm}$. При отождествлении многообразий $\mathbb{R} V_{ \pm}$с топологическими многообразиями $M_{ \pm}$вещественные исчезающие сферы $\Sigma_{ \pm}^{\tau}$ отождествляются с окружностями

$$
\begin{gathered}
S_{+}^{1}:\left\{\begin{array}{l}
u_{1}^{2}+u_{2}^{2}=r^{2} \\
u_{3}=u_{4}=0,
\end{array}\right. \\
S_{-}^{1}:\left\{\begin{array}{l}
u_{1}=u_{2}=0 \\
u_{3}^{2}+u_{4}^{2}=r^{2} .
\end{array}\right.
\end{gathered}
$$

Необходимо показать, что один из классов гомологий

$$
\left[\Sigma_{+}^{\tau}\right] \in H_{1}\left(\mathbb{R} V_{+}, \mathbb{F}_{2}\right), \quad\left[\Sigma_{-}^{\tau}\right] \in H_{1}\left(\mathbb{R} V_{-}, \mathbb{F}_{2}\right)
$$

равен нулю, а другой не равен. Если применить произведенные отождествления, то достаточно проверить, что один из классов гомологий

$$
\left[S_{+}^{1}\right] \in H_{1}\left(M_{+}, \mathbb{F}_{2}\right), \quad\left[S_{-}^{1}\right] \in H_{1}\left(M_{-}, \mathbb{F}_{2}\right)
$$

равен нулю, а другой не равен. Введем еще две окружности на крае многообразия $M$

$$
\begin{aligned}
& s_{1}:\left\{\begin{array}{l}
u_{1}^{2}+u_{2}^{2}=r^{2} \\
u_{3}=r, u_{4}=0,
\end{array}\right. \\
& s_{2}:\left\{\begin{array}{l}
u_{1}=r, u_{2}=0 \\
u_{3}^{2}+u_{4}^{2}=r^{2} .
\end{array}\right.
\end{aligned}
$$

Эти окружности лежат на торе $T$ и задают образующие группы $H_{1}\left(T, \mathbb{F}_{2}\right)$. Тор $T$ является общей границей полноториев $T_{ \pm}$, причем окружность $s_{1}$ является параллелью полнотория $T_{+}$и меридианом полнотория $T_{-}$, а окружность $s_{2}$ является параллелью полнотория $T_{-}$и меридианом полнотория $T_{+}$. Поэтому равенство нулю класса гомологий $\left[S_{+}^{1}\right] \in H_{1}\left(M_{+}, \mathbb{F}_{2}\right)$ равносильно равенству нулю класса гомологий $\left[s_{1}\right] \in H_{1}\left(M, \mathbb{F}_{2}\right)$, а равенство нулю класса гомологий $\left[S_{-}^{1}\right] \in H_{1}\left(M_{+}, \mathbb{F}_{2}\right)$ равносильно равенству нулю класса гомологий $\left[s_{2}\right] \in H_{1}\left(M, \mathbb{F}_{2}\right)$. Следовательно, нам достаточно показать, что один из классов гомологий $\left[s_{1}\right],\left[s_{2}\right] \in H_{1}\left(M, \mathbb{F}_{2}\right)$ равен нулю, а другой не равен. Тогда соответственно один из классов гомологий

$$
\left[S_{+}^{1}\right] \in H_{1}\left(M_{+}, \mathbb{F}_{2}\right), \quad\left[S_{-}^{1}\right] \in H_{1}\left(M_{-}, \mathbb{F}_{2}\right)
$$

будет равен нулю, а другой не равен. Для проверки этого утверждения рассмотрим длинную точную гомологическую последовательность пары $(M, T)$

$$
0 \rightarrow H_{2}\left(M, \mathbb{F}_{2}\right) \rightarrow H_{2}\left(M, T ; \mathbb{F}_{2}\right) \rightarrow H_{1}\left(T, \mathbb{F}_{2}\right) \rightarrow H_{1}\left(M, \mathbb{F}_{2}\right) \rightarrow \ldots
$$

Заметим теперь, что имеют место равенства

$$
\begin{aligned}
\operatorname{dim} H_{2}\left(M, T ; \mathbb{F}_{2}\right) & =\operatorname{dim} H_{2}\left(\mathbb{R} V_{0},\left\{p_{0}\right\} ; \mathbb{F}_{2}\right) \\
& =\operatorname{dim} H_{2}\left(\mathbb{R} V_{0}, \mathbb{F}_{2}\right)=\operatorname{dim} H_{2}\left(\mathbb{R} \tilde{Y}, \mathbb{F}_{2}\right)=6,
\end{aligned}
$$


а так как класс гомологий $[\mathbb{R} \widetilde{Q}] \in H_{2}\left(\mathbb{R} \tilde{Y}, \mathbb{F}_{2}\right)$ не равен нулю, то выполняются следующие равенства:

$$
\operatorname{dim} H_{2}\left(M, \mathbb{F}_{2}\right)=\operatorname{dim} H_{2}\left(\mathbb{R} V_{0} \backslash\left\{p_{0}\right\}, \mathbb{F}_{2}\right)=\operatorname{dim} H_{2}\left(\mathbb{R} \tilde{Y} \backslash \mathbb{R} \widetilde{Q}, \mathbb{F}_{2}\right)=5 .
$$

Поэтому из точной последовательности следует, что линейно зависимы элементы $\left[s_{1}\right],\left[s_{2}\right] \in H_{1}\left(M, \mathbb{F}_{2}\right)$, причем имеется только одно соотношение. В принципе, таким соотношением может быть только одно из следуюших:

$$
\left[s_{1}\right]=0, \quad\left[s_{2}\right]=0, \quad\left[s_{1}\right]+\left[s_{2}\right]=0 .
$$

Мы должны показать, что третье соотношение не выполняется. Для доказательства этого утверждения прежде всего заметим, что имеется каноническое отображение

$$
\pi: T \rightarrow \mathbb{R} \widetilde{Q}
$$

которое является двулистным накрытием. Оно устроено следующим образом. Квадрика $\mathbb{R} \widetilde{Q}$ - это множество прямых, удовлетворяющих однородному уравнению

$$
u_{1}^{2}+u_{2}^{2}-u_{3}^{2}-u_{4}^{2}=0 .
$$

Если теперь $u \in T$, то прямая $\overline{O u}$ определяет точку $\pi(u) \in \mathbb{R} \widetilde{Q}$. Непосредственно проверяются равенства

$$
\pi_{*}\left(\left[s_{1}\right]\right)=\left[l^{\prime}\right]+\left[l^{\prime \prime}\right], \quad \pi_{*}\left(\left[s_{2}\right]\right)=\left[l^{\prime}\right]+\left[l^{\prime \prime}\right] .
$$

Заметим также, что $\pi^{-1}(\pi(u))= \pm u$, поэтому если на краю многообразия $M$, т. е. на торе $T$, произвести отождествление симметричных точек, т.е. отождествить точки $\pm u \in T$, то получим пару топологических пространств, гомеоморфную паре $(\mathbb{R} \widetilde{Y}, \mathbb{R} \widetilde{Q})$. Будем обозначать полученную пару пространств через $(\bar{M}, \bar{T})$. Заметим, что имеется канонический гомеоморфизм $\mathbb{R} \widetilde{Q} \stackrel{\approx}{\longrightarrow} \bar{T}$, который продолжается до гомеоморфизма пар $(\mathbb{R} \widetilde{Y}, \mathbb{R} \widetilde{Q}) \stackrel{\approx}{\longrightarrow}(\bar{M}, \bar{T})$. Естественное отображение пар $(M, T) \rightarrow(\bar{M}, \bar{T})$, индуцированное отождествлением точек, задает коммутативную диаграмму из точных последовательностей

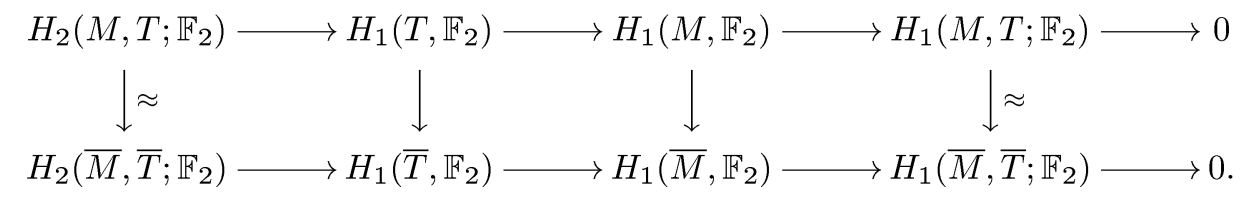

Пусть $\bar{l}^{\prime}, \bar{l}^{\prime \prime}$ - кривые на торе $\bar{T}$, которые являются образами прямых $l^{\prime}, l^{\prime \prime}$ на квадрике $\mathbb{R} \widetilde{Q}$ при каноническом отображении $\mathbb{R} \widetilde{Q} \rightarrow \bar{T}$. Тогда каждый класс гомологий $\left[s_{1}\right],\left[s_{2}\right]$ при гомоморфизме $H_{1}\left(T, \mathbb{F}_{2}\right) \rightarrow H_{1}\left(\bar{T}, \mathbb{F}_{2}\right)$ отображается в класс гомологий $\left[\bar{l}^{\prime}\right]+\left[\bar{l}^{\prime \prime}\right]$. Так как прямые $l^{\prime}, l^{\prime \prime}$ гомологичны друг другу на многообразии $\mathbb{R} \tilde{Y}$, а сами не гомологичны нулю, то класс гомологий $\left[\bar{l}^{\prime}\right]+\left[\bar{l}^{\prime \prime}\right] \in$ $H_{1}\left(\bar{M}, \mathbb{F}_{2}\right)$ равен нулю, а классы гомологий $\left[\bar{l}^{\prime}\right],\left[\bar{l}^{\prime \prime}\right] \in H_{1}\left(\bar{M}, \mathbb{F}_{2}\right)$ не равны нулю. Поэтому класс гомологий $\left[\bar{l}^{\prime}\right]+\left[\bar{l}^{\prime \prime}\right] \in H_{1}\left(\bar{T}, \mathbb{F}_{2}\right)$ лежит в образе гомоморфизма $H_{2}\left(\bar{M}, \bar{T} ; \mathbb{F}_{2}\right) \rightarrow H_{1}\left(\bar{T}, \mathbb{F}_{2}\right)$. Тогда из коммутативной диаграммы вытекает, что класс гомологий $\left[s_{1}\right]+\left[s_{2}\right] \in H_{1}\left(T, \mathbb{F}_{2}\right)$ не может лежать в образе гомоморфизма $H_{2}\left(M, T ; \mathbb{F}_{2}\right) \rightarrow H_{1}\left(T, \mathbb{F}_{2}\right)$, следовательно, соотношение $\left[s_{1}\right]+\left[s_{2}\right]=0$ не выполняется. Предложение доказано. 
ЗАмЕчАниЕ 3.6. Из доказательства предложения 3.5 видно, когда при выполнении условий предложения кубика $\left(V_{+}, \tau\right)$ будет $M$-многообразием, а именно справедливы следуюшие утверждения:

1) если квадрика $\mathbb{R} Q$ - эллипсоид и исчезаюшая сфера $\Sigma_{+}^{\tau}$ двумерная, то кубика $\left(V_{+}, \tau\right)$ всегда будет $M$-многообразием;

2) если квадрика $\mathbb{R} Q$ - гиперболоид и исчезающая окружность $\Sigma_{+}^{\tau}$ не гомологична нулю, то кубика $\left(V_{+}, \tau\right)$ будет $M$-многообразием; исчезающая окружность $\Sigma_{+}^{\tau}$ не гомологична нулю, если топологическое многообразие $V_{+}$получается из $V_{0}$ заменой конуса $K$ на полноторие $T_{+}$таким образом, что окружность $s_{2}$, гомологичная нулю на многообразии с краем $M=V_{0} \backslash \stackrel{\circ}{K}$, становится меридианом полнотория $T_{+}$.

В основе доказательства теоремы 0.2 лежит описание устройства вещественной части $\mathbb{R} F_{0}$ поверхности $\Phi$ ано особой кубики, изложенное в $\S 2$. Согласно этому описанию поверхность $\mathbb{R} F_{0}$ получается из поверхности $\mathbb{R} C^{(2)}$ склейкой кривых $\mathbb{R} C^{\prime}, \mathbb{R} C^{\prime \prime}$ с помощью диффеоморфизма $\lambda_{\mathbb{R}}^{\prime \prime} \circ\left(\lambda_{\mathbb{R}}^{\prime}\right)^{-1}$. Предложение 2.3 показывает, какая после склейки получается поверхность. Из семейства кубик $V_{t}$ получается семейство поверхностей Фано $F_{t}$. В [3] показано, что полное тело, составленное из этих поверхностей, т. е. множество

$$
\mathfrak{F}=\left\{(L, t) \in F_{Z} \times \mathbb{P}^{1}: L \subset V_{t}\right\},
$$

будет неособым многообразием. Здесь $F_{Z}$ - многообразие Фано, параметризующее прямые на четырехмерной кубике $Z$.

Кривые $C^{\prime}, C^{\prime \prime} \subset C^{(2)}$ не пересекаются, а классы гомологий $\left[C^{\prime}\right],\left[C^{\prime \prime}\right] \in$ $H_{2}\left(C^{(2)}, \mathbb{Z}\right)$ равны в силу следствия 1.4 . Обозначим $\pi^{\prime}: N^{\prime} \rightarrow C^{\prime}, \pi^{\prime \prime}: N^{\prime \prime} \rightarrow C^{\prime \prime}$ нормальные комплексные расслоения к кривым $C^{\prime}, C^{\prime \prime}$ в $C^{(2)}$. Тогда эти расслоения тривиальны в категории дифференцируемых комплексных векторных расслоений, так как индексы пересечений $\left(C^{\prime}, C^{\prime}\right)=\left(C^{\prime \prime}, C^{\prime \prime}\right)=\left(C^{\prime}, C^{\prime \prime}\right)$ равны нулю. Обозначим через $N_{C}$ нормальное расслоение к $C$ в $\mathfrak{F}$ и покажем, что вьполняется равенство

$$
N_{C}=\left(\lambda^{\prime}\right)^{*}\left(N^{\prime}\right) \oplus\left(\lambda^{\prime \prime}\right)^{*}\left(N^{\prime \prime}\right) .
$$

Расслоения $\left(\lambda^{\prime}\right)^{*}\left(N^{\prime}\right),\left(\lambda^{\prime \prime}\right)^{*}\left(N^{\prime \prime}\right)$ будем обозначать через $N_{C}^{\prime}, N_{C}^{\prime \prime}$ соответственно, тогда предыдушее равенство запишется следующим образом:

$$
N_{C}=N_{C}^{\prime} \oplus N_{C}^{\prime \prime}
$$

Пусть $U^{\prime}, U^{\prime \prime}$ - замкнутые окрестности кривых $C^{\prime}, C^{\prime \prime}$ в $C^{(2)}$. Образы этих окрестностей при отображении $\lambda: C^{(2)} \rightarrow F_{0}$ обозначим через $U_{C}^{\prime}, U_{C}^{\prime \prime}$ соответственно и назовем ветвям $и$ поверхности $F_{0}$. Заметим, что $C$ - это кривая самопересечения особой поверхности $F_{0}$, через эту кривую проходят трансверсально две неособые ветви $U_{C}^{\prime}, U_{C}^{\prime \prime}$ поверхности $F_{0}$, а расслоения $N_{C}^{\prime}, N_{C}^{\prime \prime}$ являются нормальными расслоениями к кривой $C$ на этих ветвях. Эти ветви трансверсально пересекаются в $\mathfrak{F}$ по кривой $C$, поэтому выполняется равенство (3.1). Расслоение $N_{C}$ тривиально в категории дифференцируемых комплексных векторных расслоений, 
так как расслоения $N_{C}^{\prime}, N_{C}^{\prime \prime}$ тривиальны. На расслоении $N_{C}$ имеется вещественная структура $\varphi: N_{C} \rightarrow N_{C}$. Это антилинейная инволюция, которая индуцирована антилинейной инволюцией касательных расслоений $\tau_{*}: T \mathfrak{F} \rightarrow T \mathfrak{F}$, для нее коммутативна диаграмма

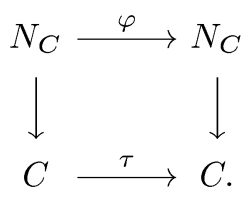

Таким образом, пара $\left(N_{C}, \varphi\right)$ является вещественным расслоением в терминологии статьи [2]. Через $\mathbb{R} N_{C}$ обозначим вешественную часть расслоения $N_{C}$, т. е. множество неподвижных точек расслоения $N_{C}$ относительно инволюции $\varphi$. Тогда $\mathbb{R} N_{C}$ является нормальным расслоением к кривой $\mathbb{R} C$ на теле $\mathbb{R} \mathfrak{F}$.

ЛЕмма 3.7. Расслоение $\mathbb{R} N_{C}$ является тривиальным расслоением в категории дифференцируемых вещественных расслоений.

ДокАЗАТЕЛьство. В случае, когда квадрика $\mathbb{R} Q$ является гиперболоидом, утверждение леммы следует из равенства

$$
\mathbb{R} N_{C}=\mathbb{R} N_{C}^{\prime} \oplus \mathbb{R} N_{C}^{\prime \prime}
$$

и тривиальности расслоений $\mathbb{R} N_{C}^{\prime}, \mathbb{R} N_{C}^{\prime \prime}$, тривиальность которых устанавливается так же, как была установлена тривиальность расслоений $N_{C}^{\prime}, N_{C}^{\prime \prime}$. Действительно, кривые $\mathbb{R} C^{\prime}, \mathbb{R} C^{\prime \prime} \subset \mathbb{R} C^{(2)}$ не пересекаются, а классы гомологий $\left[\mathbb{R} C^{\prime}\right]$, $\left[\mathbb{R} C^{\prime \prime}\right] \in H_{2}\left(\mathbb{R} C^{(2)}, \mathbb{F}_{2}\right)$ равны в силу предложения 1.9 .

В случае, когда квадрика $\mathbb{R} Q$ является эллипсоидом, мы будем доказьвать, что даже вещественное расслоение $\left(N_{C}, \varphi\right)$ тривиально. Для этого нужно показать, что расслоение $\left(N_{C}, \varphi\right)$ изоморфно расслоению $\left(C \times \mathbb{C}^{2}, \varphi\right)$, на котором инволюция

$$
\varphi: C \times \mathbb{C}^{2} \rightarrow C \times \mathbb{C}^{2}
$$

задается правилом $\varphi(p, z)=(\tau(p), \bar{z})$, черта означает комплексное сопряжение. Касательное отображение $\tau_{*}: T \mathfrak{F} \rightarrow T \mathfrak{F}$ переводит расслоение $N_{C}^{\prime}$ на расслоение $N_{C}^{\prime \prime}$, а также расслоение $N_{C}^{\prime \prime}$ на расслоение $N_{C}^{\prime}$. Выберем какую-нибудь тривиализацию $\nu^{\prime}: N_{C}^{\prime} \rightarrow C \times \mathbb{C}$. Пусть $\theta: C \times \mathbb{C} \rightarrow C \times \mathbb{C}$ - антилинейное отображение, заданное правилом $\theta(p, z)=(\tau(p), \bar{z})$, где черта означает комплексное сопряжение. Тогда в качестве тривиализации расслоения $N_{C}^{\prime \prime}$ возьмем отображение $\nu^{\prime \prime}: N_{C}^{\prime \prime} \rightarrow C \times \mathbb{C}$, где $\nu^{\prime \prime}=\theta \circ \nu^{\prime} \circ \tau_{*}$. Положим теперь $\nu=\sigma \circ\left(\nu^{\prime} \oplus \nu^{\prime \prime}\right)$, где отображение $\sigma: C \times \mathbb{C}^{2} \rightarrow C \times \mathbb{C}^{2}$ задается правилом $\sigma\left(p,\left(z_{1}, z_{2}\right)\right)=\left(p,\left(z_{2}, z_{1}\right)\right)$, т. е. переставляет координаты. Тогда непосредственно проверяется, что отображение $\nu: N_{C} \rightarrow C \times \mathbb{C}^{2}$ является искомой тривиализацией. Лемма доказана.

ЗАмЕчАниЕ 3.8. Вещественное расслоение $\left(N_{C}, \varphi\right)$ тривиально и в случае, когда квадрика $\mathbb{R} Q$ является гиперболоидом.

Из леммы 3.7 вытекает, что замкнутая окрестность кривой $\mathbb{R} C$ на теле $\mathbb{R} \mathfrak{F}$ представляет собой произведение $\mathbb{R} C \times D$, где $D=\left\{x^{2}+y^{2} \leqslant r\right\}$ - двумерный замкнутьй диск. Окрестность $\mathbb{R} C \times D$ кривой $\mathbb{R} C$ является объединением непересекаюшихся окрестностей $\Gamma_{i} \times D$ кривых $\Gamma_{i}, i=0, \ldots, 4$. Рассмотрим функцию 
$f: \mathbb{R} \mathfrak{F} \rightarrow \mathbb{R P}^{1}$, заданную правилом $\mathbb{R} \mathfrak{F} \ni(L, t) \mapsto t \in \mathbb{R} \mathbb{P}^{1}$. Ее ограничение на диск $D_{p}=p \times D$, где $p \in \mathbb{R} C$, имеет единственную невырожденную критическую точку $p=(p, 0)$. Можно выбрать такую замкнутую окрестность кривой $\Gamma_{i}$ и построить на ней такую структуру прямого произведения $\Gamma_{i} \times D$, что функция $f$ на этой окрестности примет вид $f= \pm x^{2} \pm y^{2}$.

Перейдем непосредственно к доказательству теоремы 0.2. Сначала рассмотрим случай, когда квадрика $\mathbb{R} Q$ является эллипсоидом. Из первой части предложения 2.3 следует, что критическая точка $p=(p, 0) \in D_{p}$ функции $\left.f\right|_{D_{p}}$ является точкой минимума или максимума. Поэтому для всех точек $p \in \Gamma_{i}$ при фиксированном номере $i=0, \ldots, 4$ пересечение

$$
f^{-1}(\varepsilon) \cap D_{p}=\mathbb{R} F_{\varepsilon} \cap D_{p}
$$

должно быть или окружностью, или пустым множеством. В первом случае пересечение

$$
f^{-1}(\varepsilon) \cap \Gamma_{i} \times D=\mathbb{R} F_{\varepsilon} \cap \Gamma_{i} \times D
$$

представляет собой тор, а во втором случае это пересечение является пустым множеством. Таким образом, особая поверхность $\mathbb{R} F_{0}=\mathbb{R} C^{(2)} \cup \mathbb{R} C$ перестраивается в неособую поверхность $\mathbb{R} F_{\varepsilon}$ следуюшим образом: компоненты из $\mathbb{R} C^{(2)}$ не меняются, а каждая кривая $\Gamma_{i} \subset \mathbb{R} C$ или заменяется на тор, или исчезает. Поскольку особая и неособая поверхности Фано $\left(F_{0}, \tau\right),\left(F_{\varepsilon}, \tau\right)$ являются $M$-поверхностями, то выполняются равенства

$$
\begin{aligned}
& \operatorname{dim} H_{*}\left(\mathbb{R} F_{0}, \mathbb{F}_{2}\right)=\operatorname{dim} H_{*}\left(F_{0}, \mathbb{F}_{2}\right)=57, \\
& \operatorname{dim} H_{*}\left(\mathbb{R} F_{\varepsilon}, \mathbb{F}_{2}\right)=\operatorname{dim} H_{*}\left(F_{\varepsilon}, \mathbb{F}_{2}\right)=67 .
\end{aligned}
$$

Следовательно, перестройки должны повысить размерность $\operatorname{dim} H_{*}\left(\mathbb{R} F_{0}, \mathbb{F}_{2}\right)$ на 10, а это возможно, только если все перестройки заменяют кривые $\Gamma_{i}, \quad i=$ $0, \ldots, 4$, на торы. Поэтому поверхность $\mathbb{R} F$ имеет следующий топологический тип: $\mathbb{R} C^{(2)} \sqcup 5 T^{2}$. Остается заметить, что поверхность $\mathbb{R} C^{(2)}$ гомеоморфна поверхности ${ }_{5} S^{2} \sqcup 5 T^{2}$

Рассмотрим теперь случай, когда квадрика $\mathbb{R} Q$ является гиперболоидом. В этом случае перестройка особой поверхности $\mathbb{R} F_{0}$ в неособую поверхность $\mathbb{R} F_{\varepsilon}$ происходит следующим образом. Пересечение $\mathbb{R} F_{0} \cap \Gamma_{i} \times D$ представляет собой произведение кривой $\Gamma_{i}$ на крест $\left\{x^{2}-y^{2}=0\right\} \cap D$. Это произведение заменяется на произведение кривой $\Gamma_{i}$ и двух дуг гиперболы $\left\{x^{2}-y^{2}= \pm \varepsilon\right\} \cap D$. Поэтому все торы $T_{i j}$ при $1 \leqslant i<j \leqslant 4$ не меняются. Особая поверхность $\bar{T}_{0 i}$ с трансверсальным самопересечением по кривой $\Gamma_{i}, i=1, \ldots, 4$, может перестроиться двумя способами: или два тора $T_{i}^{\prime}, T_{i}^{\prime \prime}$ преобразуются в один тор $T_{0 i}$, или расходятся и становятся непересекающимися. Эти преобразования назовем соответственно перестройками первого и второго типа. Аналогичные перестройки возможны с особой поверхностью $\bar{\Pi}$, которая имеет самопересечение по кривой $\Gamma_{0}$ : или две поверхности $\Pi_{1}, \Pi_{2}$ преобразуются в одну поверхность П, или поверхности $\Pi_{1}, \Pi_{2}$ расходятся и становятся непересекающимися. Но так как поверхности Фано $\left(F_{0}, \tau\right),\left(F_{\varepsilon}, \tau\right)$ являются $M$-поверхностями, то возможны только перестройки второго типа. Поэтому поверхность $\mathbb{R} F_{\varepsilon}$ имеет топологический тип ${ }_{5} S^{2} \sqcup 15 T^{2}$. Теорема 0.2 полностью доказана. 


\section{Список литературы}

1. Арнольд В.И., Варченко А.Н., Гусейн-Заде С.М. Особенности дифференцируемых отображений. Монодромия и асимптотика интегралов. М.: Наука, 1984.

2. Atiyah M.F. K-theory and reality // Quart. J. Math. Oxford. 1966. V. 17. № 2. P. $165-193$

3. Clemens C. H., Griffits P.A. The intermediate Jacobian of the cubic threefold // Ann. Math. 1972. V. 95. P. 281-356.

4. Degtyarev A., Itenberg I., Kharlamov V. Real Enriques surfaces // Lect. Not. Math. V. 1746. N. Y.: Springer-Verlag, 2000.

5. Дегтярев А.И., Звонилов В.И. Жесткая изотопическая классификация вещественных алгебраических кривых бистепени $(3,3)$ на квадриках // Матем. заметки. 1999. T. 66. № 6. C. $810-815$.

6. Гротендик A. О некоторых вопросах гомологической алгебры. М.: ИЛ, 1961.

7. Краснов В.А. Неравенства Гарнака-Тома для отображений вещественных алгебраических многообразий // Изв. АН СССР. Сер. матем. 1983. Т. 47. № 2. С. 268-297.

8. Краснов В.А. Вещественные алгебраические GM-многообразия // Изв. РАН. Сер. матем. 1998. Т. 62. № 3. С. 39-66.

9. Краснов В.А. О поверхности Фано вещественной трехмерной $M$-кубики // Матем. заметки. 2005. Т. 78. № 5. С. 710-717.

10. Милнор Дж. Теория Морса. М.: Мир, 1965.

11. Милнор Дж. Особые точки комплексных гиперповерхностей. М.: Мир, 1971.

Ярославский государственный университет

Поступило в редакцию

E-mail: krasnov@uniyar.ac.ru

27.01.2005 